\title{
Oxidative Aromatization of Dihydropyrimidines and Dihydropyrimidinones
}

\author{
Kana Yamamoto,* Ye Grace Chen, Frédéric Buono \\ Process Research and Development \\ Bristol-Myers Squibb Pharmaceutical Institute \\ One Squibb Drive, New Brunswick, New Jersey 08903
}

\section{Supporting Information}

\section{General Information.}

All reactions were performed under nitrogen atmosphere. Solvents were used as purchased (EM Science) without further purification. Flash chromatography was performed with silica gel 60 (230-400 mesh) using an ISCO automated chromatography apparatus. Analytical thin layer chromatography was performed on EM Reagent $0.25 \mathrm{~mm}$ silica gel 60-F plates, and visualization was accomplished with UV light $(254 \mathrm{~nm})$. Infrared spectra were recorded on a Protégé 460 Magna-IR Technology spectrometer either in $\mathrm{KBr}$ pellets or as thin film on a $\mathrm{NaCl}$ tablet. ${ }^{1} \mathrm{H}$ - and ${ }^{13} \mathrm{C}-\mathrm{NMR}$ spectra were recorded on a Bruker AC-300 spectrometer $\left({ }^{1} \mathrm{H} ; 300 \mathrm{MHz},{ }^{13} \mathrm{C} ; 75 \mathrm{MHz}\right)$ and are reported in ppm using solvent as an internal standard (DMSO: ${ }^{1} \mathrm{H} ; 2.49,{ }^{13} \mathrm{C} ; 39.5 . \mathrm{CDCl}_{3}{ }^{1} \mathrm{H} ; 7.25,{ }^{13} \mathrm{C} ; 77.0$ ). 2-D NMR experiments were performed on Bruker DRX600 spectrometer $\left({ }^{1} \mathrm{H} ; 600.13 \mathrm{MHz},{ }^{13} \mathrm{C} ; 150.9 \mathrm{MHz}\right)$. Low resolution mass spectra were obtained on HPLC/MS/MS (Shimadzu HPLC equipped with Waters ZQ mass spectrometer). High resolution mass spectra were obtained on Micromass QTOF API US with lockspray (Lockmass: Enkephalin, $\mathrm{MH}^{+}$, 556.2771). Analytical HPLC was routinely performed for reaction monitoring with the following conditions: column = YMC-pack Pro C18 $4.6 \times 50 \mathrm{~mm}^{2}$ S-3 $\mu \mathrm{m}$, flow rate $=2 \mathrm{~mL} / \mathrm{min}$, detector $220 \mathrm{~nm}$, injection volume 5-10 $\mu \mathrm{L}$, column temperature $=$ rt. Mobile phase weak solvent (A): $0.05 \%$ TFA in $\mathrm{MeCN}: \mathrm{H}_{2} \mathrm{O}$ (10:90), strong solvent (B): 0.05\% TFA in $\mathrm{MeCN}: \mathrm{H}_{2} \mathrm{O}$ (95:5). Gradient program $=$ start: $5 \%$ solvent $\mathrm{B}$, final: $100 \%$ solvent $\mathrm{B}$, gradient time $=8 \mathrm{~min}$, stop time $=10 \mathrm{~min}$.

\section{General procedure for the Biginelli three-component-coupling for the preparation of compounds 1a-1j.}

Compounds $\mathbf{1 a - 1 j}$ were prepared according to the literature. ${ }^{1}$ The product obtained with this procedure contained $\sim 10-20$ ppm copper. A typical procedure is as the following:

A round bottom flask was charged with methanol (1 1), 4-fluorobenzaldehyde (124 g, 1 mole, 1 equiv), methyl isobutyrylacetate (144 g, 1 mole, 1 equiv) and urea (105 g, 1.75 mole, 1.75 equiv). To the mixture was added copper (I) chloride (1.01 g, 0.01 mole, 0.01 equiv) and conc. (95-97\%) sulfuric acid (10 ml) and the batch was heated to refluxing temperature $\left(65-67^{\circ} \mathrm{C}\right)$. The solution became heterogeneous within 2-3 h. After heating at reflux for $24 \mathrm{~h}$, the mixture was cooled to rt $\left(20-25^{\circ} \mathrm{C}\right)$ and the product was isolated by filtration. The wet cake was washed once with methanol and was dried under reduced pressure (30 torr) at $40{ }^{\circ} \mathrm{C}$ for $10 \mathrm{~h}$. Dihydropyrimidinone (1i) was obtained as a white granular solid (182 g, 0.62 mole, 62\%).

\footnotetext{
1 Matsushima, A.; Oda, M.; Kawachi, Y.; Chika, J. PCT WO 03/006439 A1.
} 


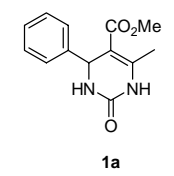

5-Methoxycarbonyl-6-methyl-4-phenyl-3,4-dihydropyrimidin-2(1H)-one (1a). ${ }^{2}$ Yield: 76\%. ${ }^{1} \mathrm{H}-\mathrm{NMR}$ and LRMS data matched with those reported in the literature. In order to obtain a strictly metal-free product, this compound was also prepared without any metal catalyst ( 5 mol\% p-toluenesulfonic acid was used in place of copper chloride) which gave $58 \%$ yield. ${ }^{1} \mathrm{H}-\mathrm{NMR}\left(300 \mathrm{MHz}, d_{6}\right.$-DMSO): $\delta 2.24(\mathrm{~s}, 3 \mathrm{H}) ; 3.52(\mathrm{~s}, 3 \mathrm{H}) ; 5.14(\mathrm{~d}, 1 \mathrm{H}, J=3.1) ; 7.22(\mathrm{~m}, 3 \mathrm{H}) ; 7.30(\mathrm{~m}$, $2 \mathrm{H}) ; 7.75(\mathrm{~s}, 1 \mathrm{H}) ; 9.21(\mathrm{~s}, 1 \mathrm{H}) .{ }^{13} \mathrm{C}-\mathrm{NMR}\left(75 \mathrm{MHz}, d_{6}\right.$-DMSO): $\delta 17.8,50.8,53.8,99.0,126.2,127.3,128.5,144.7$, 148.7, 152.2, 165.8. ESIMS m/z $247\left(\left[\mathrm{M}+\mathrm{H}^{+}\right], \mathrm{C}_{13} \mathrm{H}_{14} \mathrm{~N}_{2} \mathrm{O}_{3}\right.$ requires 246).

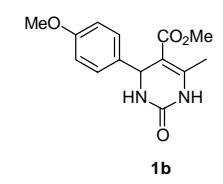

5-Methoxycarbonyl-4-(4'-methoxy)phenyl-6-methyl-3,4-dihydropyrimidin-2(1H)-one (1b). ${ }^{2}$ Yield: 82\%. ${ }^{1} \mathrm{H}-\mathrm{NMR}(300$ MHz, $d_{6}$-DMSO): $\delta 2.24(\mathrm{~s}, 3 \mathrm{H}) ; 3.51(\mathrm{~s}, 3 \mathrm{H}) ; 3.71(\mathrm{~s}, 3 \mathrm{H}) ; 5.09(\mathrm{~s}, 1 \mathrm{H}) ; 6.87(\mathrm{~d}, 2 \mathrm{H}, J=8.5) ; 7.14(\mathrm{~d}, 1 \mathrm{H}, J=8.4) ; 7.68$ $(\mathrm{s}, 1 \mathrm{H}) ; 9.18$ (s. $1 \mathrm{H}) .{ }^{13} \mathrm{C}-\mathrm{NMR}\left(75 \mathrm{MHz}, d_{6}\right.$-DMSO): $\delta 17.8,50.7,53.2,55.0,99.3,113.8,127.3,136.9,148.3,152.2$, 158.5, 165.9. ESIMS m/z $277\left(\left[\mathrm{M}+\mathrm{H}^{+}\right], \mathrm{C}_{13} \mathrm{H}_{16} \mathrm{~N}_{2} \mathrm{O}_{4}\right.$ requires 276).

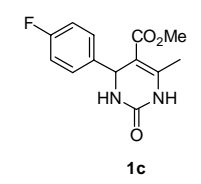

4-(4'-Fluoro)phenyl 5-methoxycarbonyl-6-methyl-3,4-dihydropyrimidin-2(1H)-one (1c). ${ }^{3}$ Yield: 85\%. ${ }^{1} \mathrm{H}-\mathrm{NMR}(300$ MHz, $d_{6}$-DMSO): $\delta 2.24(\mathrm{~s}, 3 \mathrm{H}) ; 3.52(\mathrm{~s}, 3 \mathrm{H}) ; 5.14(\mathrm{~d}, 1 \mathrm{H}, J=3.0) ; 7.13(\mathrm{dd}, 2 \mathrm{H}, J=8.7,8.8) ; 7.26(\mathrm{dd}, 1 \mathrm{H}, J=5.7,8.5)$; 7.75 (s, 1H); 9.23 (s. 1H). ${ }^{13} \mathrm{C}-\mathrm{NMR}\left(75 \mathrm{MHz}, d_{6}\right.$-DMSO): $\delta 17.8,50.8,53.1,98.9,115.2(\mathrm{~d}, \mathrm{~J}=22), 128.6(\mathrm{~d}, J=8.4)$; 140.9, 148.8, 152.0, $161.3(\mathrm{~d}, J=243)$, 165.7. ESIMS m/z $265\left(\left[\mathrm{M}+\mathrm{H}^{+}\right], \mathrm{C}_{13} \mathrm{H}_{13} \mathrm{FN}_{2} \mathrm{O}_{3}\right.$ requires 264).

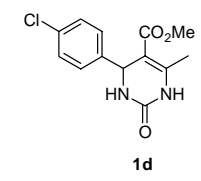

4-(4'-chloro)phenyl-5-methoxycarbonyl-6-methyl-3,4-dihydropyrimidin-2(1H)-one (1d). ${ }^{2}$ Yield: $81 \% .{ }^{1} \mathrm{H}-\mathrm{NMR}(300$ MHz, $d_{6}$-DMSO): $\delta 2.24$ (s, 3H); $3.52(\mathrm{~s}, 3 \mathrm{H}) ; 5.12(\mathrm{~d}, 1 \mathrm{H}, J=3.4) ; 7.23(\mathrm{~d}, 2 \mathrm{H}, J=8.5) ; 7.38(\mathrm{~d}, 1 \mathrm{H}, J=8.4) ; 7.79$ (s, $1 \mathrm{H}) ; 9.27$ (s. 1H). ${ }^{13} \mathrm{C}-\mathrm{NMR}\left(75 \mathrm{MHz}, d_{6}\right.$-DMSO): $\delta$ 17.9, 50.9, 53.2, 98.6, 128.1, 128.5, 131.8, 143.6, 149.0, 152.0, 165.7. ESIMS m/z $281\left(\left[\mathrm{M}+\mathrm{H}^{+}\right], \mathrm{C}_{13} \mathrm{H}_{13} \mathrm{ClN}_{2} \mathrm{O}_{3}\right.$ requires 280).

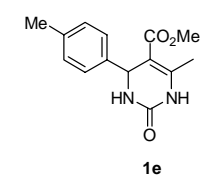

2 Hu, E. H.; Sidler, D. R.; Dolling, U-H. J. Org. Chem. 1998, 63, 3454. 
5-Methoxycarbonyl-6-methyl-4-(4'-methyl)phenyl-3,4-dihydropyrimidin-2(1H)-one (1e). ${ }^{4}$ Yield: $75 \% . \quad{ }^{1} \mathrm{H}-\mathrm{NMR}$ and LRMS data matched with those reported in the literature. ${ }^{1} \mathrm{H}-\mathrm{NMR}\left(300 \mathrm{MHz}, d_{6}\right.$-DMSO): $\delta 2.23$ (s, 3H); 2.25 (s, 3H); $3.51(\mathrm{~s}, 3 \mathrm{H}) ; 5.09(\mathrm{~d}, 1 \mathrm{H}, J=3.4) ; 7.10(\mathrm{~s}, 4 \mathrm{H}) ; 7.71(\mathrm{~s}, 1 \mathrm{H}) ; 9.19(\mathrm{~s} .1 \mathrm{H}) .{ }^{13} \mathrm{C}-\mathrm{NMR}\left(75 \mathrm{MHz}, d_{6}\right.$-DMSO): $\delta 17.9,50.9$, 53.2, 98.6, 128.1, 128.5, 131.8, 143.6, 149.0, 152.0, 165.7. IR (KBr) $v_{\max } 1093,1274,1467,1651,1700,3221,3335 \mathrm{~cm}^{-1}$. ESIMS m/z $261\left(\left[\mathrm{M}+\mathrm{H}^{+}\right], \mathrm{C}_{14} \mathrm{H}_{16} \mathrm{~N}_{2} \mathrm{O}_{3}\right.$ requires 260). Analytical calculated for $\mathrm{C}_{14} \mathrm{H}_{16} \mathrm{~N}_{2} \mathrm{O}_{3}: \mathrm{C}, 64.60 ; \mathrm{H}, 6.19 ; \mathrm{N}, 10.76$. Found: C, 64.63, H, 6.09; N, 10.61 .

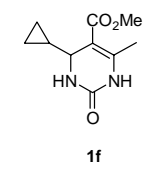

4-Cyclopropyl-5-methoxycarbonyl-6-methyl-3,4-dihydropyrimidin-2(1H)-one (1f). Yield: 48\%. ${ }^{1} \mathrm{H}-\mathrm{NMR}\left(300 \mathrm{MHz}, d_{6^{-}}\right.$ DMSO): $\delta 0.17(\mathrm{~m}, 2 \mathrm{H}) ; 0.30(\mathrm{~m}, 3 \mathrm{H}) ; 0.93(\mathrm{~m}, 2 \mathrm{H}) ; 2.16(\mathrm{~s}, 3 \mathrm{H}) ; 3.59$ (s, 3H); $3.68(\mathrm{~d}, 1 \mathrm{H}, J=3.3) ; 7.29$ (s, $1 \mathrm{H}) ; 9.00(\mathrm{~s}$, 1H). ${ }^{13} \mathrm{C}-\mathrm{NMR}$ (75 MHz, $d_{6}$-DMSO): $\delta$ 1.23, 1.77, 17.7, 50.7, 52.6, 99.1, 148.3, 12.9, 166.2. IR (KBr) $v_{\max } 1100,1235$, 1435, 1651, 1726, 3114, $3254 \mathrm{~cm}^{-1}$; ESIMS m/z $210\left(\left[\mathrm{M}+\mathrm{H}^{+}\right], \mathrm{C}_{10} \mathrm{H}_{14} \mathrm{~N}_{2} \mathrm{O}_{3} \text { requires 209). HRMS [M+H }\right]^{+}: 211.1074$ (calc, Exact mass.: 211.1083).

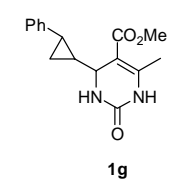

5-Methoxycarbonyl-6-methyl-4-(2'-phenyl)cyclopropyl-3,4-dihydropyrimidin-2(1H)-one (1a). The compound was purified by preparative thin-layer chromatography (70\% EtOAc/hexanes) and obtained as a mixture of (two major) diastereomers. The stereochemical relationship of the isomers was not assigned and the mixture was used directly without further purification. Yield: 17\%. ${ }^{1} \mathrm{H}-\mathrm{NMR}\left(300 \mathrm{MHz}, \mathrm{CDCl}_{3}\right): \delta 0.89(\mathrm{~m}, 3 \mathrm{H}) ; 1.14(\mathrm{~m}, 1 \mathrm{H}) ; 1.38(\mathrm{~m}, 2 \mathrm{H}) ; 1.84(\mathrm{~m}, 1 \mathrm{H})$; 2.07 (m, 1H); 2.27 (s, 3H); 2.29 (s, 3H); 3.69 (m, 3H); 3.73 (s, 3H); 4.04 (m, 2H); 6.64 (br s, 1H); 6.83 (br s, $1 \mathrm{H}) ; 6.93$ (m, 4H); $7.20(\mathrm{~m}, 6 \mathrm{H}) ; 8.79$ (br s, 2H). IR (KBr) $v_{\max } 1093,1233,1644,1702,3120,3248 \mathrm{~cm}^{-1}$. ESIMS m/z $287\left(\left[\mathrm{M}+\mathrm{H}^{+}\right]\right.$, $\mathrm{C}_{16} \mathrm{H}_{18} \mathrm{~N}_{2} \mathrm{O}_{3}$ requires 286). HRMS [M+H] $]^{+}: 287.1393$ (calc, Exact mass.: 287.1396).

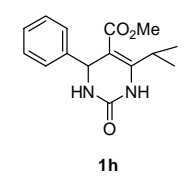

6-Isopropyl-5-methoxycarbonyl-4-phenyl-3,4-dihydropyrimidin-2(1H)-one (1h). Yield: 58\%. ${ }^{1} \mathrm{H}-\mathrm{NMR}\left(300 \mathrm{MHz}, d_{6^{-}}\right.$ DMSO): $\delta 1.12(\mathrm{~d}, 3 \mathrm{H}, J=6.6) ; 1.14(\mathrm{~d}, 3 \mathrm{H}, J=6.7) ; 3.51(\mathrm{~s}, 3 \mathrm{H}) ; 4.12(\mathrm{~m}, 1 \mathrm{H}) ; 5.13(\mathrm{~d}, 1 \mathrm{H}, J=3.4) ; 7.21-7.32(\mathrm{~m}, 5 \mathrm{H})$; $7.73(\mathrm{~s}, 1 \mathrm{H}) ; 8.87$ (s, $1 \mathrm{H}) .{ }^{13} \mathrm{C}-\mathrm{NMR}\left(75 \mathrm{MHz}, d_{6}\right.$-DMSO): $\delta 18.9,19.2,27.0,50.9,53.7,97.9,126.1,127.3,128.5,144.5$, 152.6, 152.8, 165.8. . IR (KBr) $v_{\max } 1112,1235,1629,1695 \mathrm{~cm}^{-1}$. ESIMS m/z $275\left(\left[\mathrm{M}+\mathrm{H}^{+}\right], \mathrm{C}_{15} \mathrm{H}_{18} \mathrm{~N}_{2} \mathrm{O}_{3}\right.$ requires 274).

\footnotetext{
3 Ma, Y.; Qian, C.; Wang, L.; Yang, M. J. Org. Chem. 2000, 65, 3864.

4 Fu, N-Y.; Yuan, Y-F.; Cao, Z.; Wang, A-W.; Wang, J-T.; Peppe, C. Tetrahedron 2002, $58,4801$.
} 


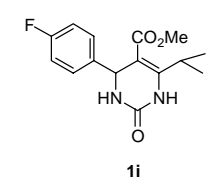

4-(4'-Fluoro)phenyl-6-isopropyl-5-methoxycarbonyl-3,4-dihydropyrimidin-2(1H)-one (1i). ${ }^{1}$ Yield: 62\%. ${ }^{1} \mathrm{H}-\mathrm{NMR}(300$ MHz, $d_{6}$-DMSO): $\delta 1.12(\mathrm{~d}, 3 \mathrm{H}, J=7.1) ; 1.14(\mathrm{~d}, 3 \mathrm{H}, J=7.0) ; 3.51(\mathrm{~s}, 3 \mathrm{H}) ; 4.12(\mathrm{~m}, 1 \mathrm{H}) ; 5.14(\mathrm{~d}, 1 \mathrm{H}, J=3.4) ; 7.14(\mathrm{dd}$, $2 \mathrm{H}, J=8.9) ; 7.25(\mathrm{dd}, 1 \mathrm{H}, J=5.7,8.9) ; 7.77(\mathrm{~s}, 1 \mathrm{H}) ; 8.92(\mathrm{~s}, 1 \mathrm{H}) .{ }^{13} \mathrm{C}-\mathrm{NMR}\left(75 \mathrm{MHz}, d_{6}\right.$-DMSO): $\delta$ 19.4, 19.6, 27.5, 51.6, 53.5, 98.5, $115.8(\mathrm{~d}, J=21) ; 128.6(\mathrm{~d}, J=8.3) ; 141.0,153.2,157.3,161.9$ (d, $J=243.2), 166.3 .{ }^{19} \mathrm{~F}-\mathrm{NMR}(282$ $\left.\mathrm{MHz}, \mathrm{CDCl}_{3}\right): \delta-115 . \tilde{8}$. IR (film) $v_{\max } 1112,1235,1629,1695 \mathrm{~cm}^{-1}$. ESIMS m/z $293\left(\left[\mathrm{M}+\mathrm{H}^{+}\right], \mathrm{C}_{15} \mathrm{H}_{17} \mathrm{FN}_{2} \mathrm{O}_{3}\right.$ requires 292). HRMS $[\mathrm{M}+\mathrm{H}]^{+}: 293.13016$ (calc, Exact mass.: 293.1302). Analytical calculated for $\mathrm{C}_{15} \mathrm{H}_{17} \mathrm{FN}_{2} \mathrm{O}_{3}: \mathrm{C}, 61.63 ; \mathrm{H}$, 5.86; N, 9.58. Found: C, 61.38, H, 5.67; N, 9.45.

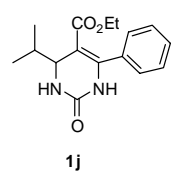

4-Isopropyl-5-methoxycarbonyl-6-phenyl-3,4-dihydropyrimidin-2(1H)-one (1j). Yield: 24\%. ${ }^{1} \mathrm{H}-\mathrm{NMR}\left(300 \mathrm{MHz}, d_{\sigma^{-}}\right.$ DMSO): $\delta 0.75(\mathrm{t}, 3 \mathrm{H}, J=7.0) ; 0.87(\mathrm{~d}, 3 \mathrm{H}, J=6.9) ; 0.90(\mathrm{~d}, 3 \mathrm{H}, J=7.2) ; 1.83(\mathrm{~m}, 1 \mathrm{H}) ; 3.74(\mathrm{q}, 2 \mathrm{H}, J=7.1) ; 4.01(\mathrm{dd}$, $1 \mathrm{H}, J=3.7,3.8) ; 7.24(\mathrm{~m}, 2 \mathrm{H}) ; 7.38(\mathrm{~m}, 3 \mathrm{H}) ; 9.00(\mathrm{~s}, 1 \mathrm{H}) .{ }^{13} \mathrm{C}-\mathrm{NMR}\left(75 \mathrm{MHz}, d_{6}\right.$-DMSO): $\delta 13.5,16.0,18.5,34.6,56.0$, 59.0, 99.3, 127.7, 128.3, 128.7, 135.5, 149.3, 153.0, 165.6. IR (KBr) $v_{\max } 1098,1249,1666,1701,3230,3309 \mathrm{~cm}^{-1}$. ESIMS m/z $289\left(\left[\mathrm{M}+\mathrm{H}^{+}\right], \mathrm{C}_{15} \mathrm{H}_{20} \mathrm{~N}_{2} \mathrm{O}_{3}\right.$ requires 288). Analytical calculated for $\mathrm{C}_{15} \mathrm{H}_{18} \mathrm{~N}_{2} \mathrm{O}_{3}: \mathrm{C}, 66.64 ; \mathrm{H}, 6.99 ; \mathrm{N}, 9.71$. Found: C, 66.58, H, 7.13; N, 9.61.

\section{General procedure for the oxidative dehydrogenation.}

A reactor was purged with nitrogen and charged with dihydropyrimidine $1 \mathbf{i}\left(700 \mathrm{~g}, 2.39\right.$ mole, 1.0 equiv), $\mathrm{CuCl}_{2}(3.25 \mathrm{~g}$, 0.024 mole, $1.0 \mathrm{~mol} \%), \mathrm{K}_{2} \mathrm{CO}_{3}\left(33.1 \mathrm{~g}, 0.243\right.$ mole, 0.1 equiv) and $\mathrm{CH}_{2} \mathrm{Cl}_{2}$ (7.0 1). The suspension was heated to $35{ }^{\circ} \mathrm{C}$ and treated with tert-butylhydroperoxide (70\% aqueous solution) (635.0 g, $677.2 \mathrm{ml}, 2.0 \mathrm{equiv})$ over $120 \mathrm{~min}$ with vigorous agitation. After $24 \mathrm{~h}, \mathrm{HPLC}$ indicated the consumption of the starting material (<3\% by relative HPLC area). The solution was cooled to $20-25{ }^{\circ} \mathrm{C}$, treated with a mixture of aqueous $\mathrm{Na}_{2} \mathrm{~S}_{2} \mathrm{O}_{3}(0.5 \mathrm{M}$ solution, 7.0 l), and $25 \mathrm{wt} / \mathrm{wt} \%$ aqueous $\mathrm{NH}_{4} \mathrm{Cl}(3.5 \mathrm{l})$, and the resulting bi-phasic mixture was stirred vigorously for $60 \mathrm{~min}$. The $\mathrm{pH}$ of the aqueous phase was $\sim 7.5-8.0$ at this point. The absence of remaining oxidant was checked by peroxide test strip. Additional thiosulfate treatment was done if necessary. The two phases were separated and the organic phase was concentrated to the minimum agitation volume via reduced pressure distillation. During this time, the product precipitated out from the solution as a white solid. A solvent exchange to heptane was performed by repeated heptane addition (2.5 1) and distillation (1-2 times) until the amount of residual $\mathrm{CH}_{2} \mathrm{Cl}_{2}$ is below $10 v / v \% \mathrm{CH}_{2} \mathrm{Cl}_{2} /$ heptane by $\mathrm{GC}$ area. The product was filtered and washed with filtrate (2.0 1), then heptane (1.0 1), and the wet cake was dried under vacuum ( 27-29 torr) at $40{ }^{\circ} \mathrm{C}$. The product $2 \mathbf{i}(666.1 \mathrm{~g}, 2.29$ mole, $96 \%)$ was obtained as a white powder. 


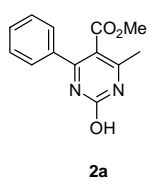

2-Hydroxy-5-methoxycarbonyl-4-methyl-6-phenylpyrimidine (2a). ${ }^{5}{ }^{1} \mathrm{H}-\mathrm{NMR}\left(300 \mathrm{MHz}, \mathrm{CDCl}_{3}\right): \delta 2.62(\mathrm{~s}, 3 \mathrm{H}) ; 3.57(\mathrm{~s}$, $3 \mathrm{H}) ; 7.46(\mathrm{~m}, 3 \mathrm{H}) ; 7.60(\mathrm{~m}, 2 \mathrm{H})$. ESIMS m/z $245\left(\left[\mathrm{M}+\mathrm{H}^{+}\right], \mathrm{C}_{13} \mathrm{H}_{12} \mathrm{~N}_{2} \mathrm{O}_{3}\right.$ requires 244).

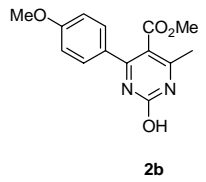

2-Hydroxy-5-methoxycarbonyl-6-(4'-methoxy)phenyl-4-methylpyrimidine (2b). ${ }^{1} \mathrm{H}-\mathrm{NMR}\left(300 \mathrm{MHz}, \mathrm{CDCl}_{3}\right): \delta 2.58$ (s, $3 \mathrm{H}) ; 3.40(\mathrm{~s}, 3 \mathrm{H}) ; 3.85(\mathrm{~s}, 3 \mathrm{H}) ; 6.95(\mathrm{~d}, 2 \mathrm{H}, J=8.9) ; 7.61$ (d, 2H, $J=8.8)$. IR (KBr) $v_{\max } 1256,1281,1434,1666,1717$, $3430 \mathrm{~cm}^{-1}$. ESIMS m/z $275\left(\left[\mathrm{M}+\mathrm{H}^{+}\right], \mathrm{C}_{14} \mathrm{H}_{14} \mathrm{~N}_{2} \mathrm{O}_{4}\right.$ requires 274). HRMS $[\mathrm{M}+\mathrm{H}]^{+}: 275.1033$ (calc, Exact mass.: 275.1032).

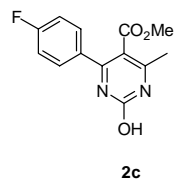

6-(4'-Fluoro)phenyl-2-hydroxy-5-methoxycarbonyl-4-methylpyrimidine (2c). ${ }^{1} \mathrm{H}-\mathrm{NMR}\left(300 \mathrm{MHz}, \mathrm{CDCl}_{3}\right): \delta 2.61(\mathrm{~s}$, $3 \mathrm{H}) ; 3.61(\mathrm{~s}, 3 \mathrm{H}) ; 7.12(\mathrm{dd}, 2 \mathrm{H}, J=8.7,8.9) ; 7.63(\mathrm{dd}, 1 \mathrm{H}, J=5.3,8.9) .{ }^{13} \mathrm{C}-\mathrm{NMR}\left(75 \mathrm{MHz}, \mathrm{CDCl}_{3}\right): \delta 52.4,77.2,111.0$, $115.6(\mathrm{~d}, J=22.0), 130.3(\mathrm{~d}, J=8.8), 158.3,166.5(\mathrm{~d}, J=253), 166.5$. IR $(\mathrm{KBr}) v_{\max } 1104,1226,1283,1434,1657,1725$, $3318 \mathrm{~cm}^{-1}$. ESIMS m/z $263\left(\left[\mathrm{M}+\mathrm{H}^{+}\right], \mathrm{C}_{13} \mathrm{H}_{11} \mathrm{FN}_{2} \mathrm{O}_{3}\right.$ requires 262). HRMS $[\mathrm{M}+\mathrm{H}]^{+}: 263.0822$ (calc, Exact mass.: 263.0832).

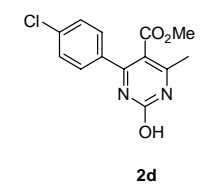

6-(4'-Chloro)phenyl-2-hydroxy-5-methoxycarbonyl-4-methylpyrimidine (2d). ${ }^{1} \mathrm{H}-\mathrm{NMR}\left(300 \mathrm{MHz}, \mathrm{CDCl}_{3}\right): \delta 2.62(\mathrm{~s}$, $3 \mathrm{H}) ; 3.63(\mathrm{~s}, 3 \mathrm{H}) ; 7.42(\mathrm{~d}, 1 \mathrm{H}, J=8.7) ; 7.57(\mathrm{~d}, 1 \mathrm{H}, J=8.5) ; 13.8$ (br s, 1H). IR (NaCl) $v_{\max } 1280,1432,1592,1657$, 1722, $3007 \mathrm{~cm}^{-1}$. ESIMS m/z $279.2\left(\left[\mathrm{M}+\mathrm{H}^{+}\right], \mathrm{C}_{13} \mathrm{H}_{11} \mathrm{ClN}_{2} \mathrm{O}_{3}\right.$ requires 278). HRMS $[\mathrm{M}+\mathrm{H}]^{+}: 279.0527$ (calc, Exact mass.: 279.0536).

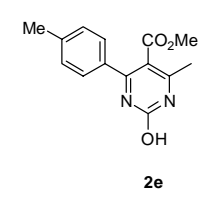

2-Hydroxy-5-methoxycarbonyl-6-(4'-methyl)phenyl-4-methylpyrimidine (2e). ${ }^{1} \mathrm{H}-\mathrm{NMR}\left(300 \mathrm{MHz}, \mathrm{CDCl}_{3}\right): \delta 2.36(\mathrm{~s}$, $3 \mathrm{H}) ; 3.32(\mathrm{~s}, 3 \mathrm{H}) ; 3.51(\mathrm{~s}, 3 \mathrm{H}) ; 7.27(\mathrm{~d}, 1 \mathrm{H}, J=8.1) ; 7.36(\mathrm{~d}, 1 \mathrm{H}, J=8.1) ; 12.3$ (br s, 1H). IR (KBr) $v_{\max } 1288,1387$,

\footnotetext{
5 Kang, F-A.; Kodah, J.; Guan, Q.; Li, X.; Murray, W. V. J. Org. Chem. 2005, 70, 1957.
} 
1433, 1601, 1656, 1731, 2747, 3007, $3420 \mathrm{~cm}^{-1}$. ESIMS m/z $259\left(\left[\mathrm{M}+\mathrm{H}^{+}\right], \mathrm{C}_{14} \mathrm{H}_{14} \mathrm{~N}_{2} \mathrm{O}_{3} \text { requires 258). HRMS [M+H }\right]^{+}$: 259.1079 (calc, Exact mass.: 259.1083).

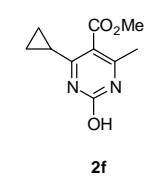

6-Cyclopropyl-2-hydroxy-5-methoxycarbonyl-4-methylpyrimidine (2f). ${ }^{1} \mathrm{H}-\mathrm{NMR}\left(300 \mathrm{MHz}, \mathrm{CDCl}_{3}\right): \delta 1.08(\mathrm{~m}, 2 \mathrm{H})$; $1.41(\mathrm{~m}, 2 \mathrm{H}) ; 2.27(\mathrm{~m}, 1 \mathrm{H}) ; 2.49$ (s, 3H); 3.91 (s, 3H). ${ }^{13} \mathrm{C}-\mathrm{NMR}\left(75 \mathrm{MHz}, \mathrm{CDCl}_{3}\right): \delta 13.7,15.9,18.7,25.7,52.4,111.8$, 157.9, 158.7, 166.3, 180.7. IR (KBr) $v_{\max } 1123,1253,1432,1592,1648,1717,3009,3400 \mathrm{~cm}^{-1}$. ESIMS m/z $209([\mathrm{M}+$ $\mathrm{H}^{+}$], $\mathrm{C}_{10} \mathrm{H}_{12} \mathrm{~N}_{2} \mathrm{O}_{3}$ requires 208). HRMS $[\mathrm{M}+\mathrm{H}]^{+}: 209.0920$ (calc, Exact mass.: 209.0926). Analytical calculated for $\mathrm{C}_{10} \mathrm{H}_{12} \mathrm{~N}_{2} \mathrm{O}_{3}$ : C, 57.68; H, 5.80; N, 13.45. Found: C, 57.28, H, 5.79; N, 13.38.

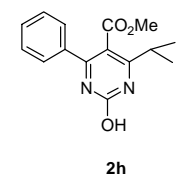

2-Hydroxy-4-isopropyl-5-methoxycarbonyl-6-phenylpyrimidine (2h). ${ }^{1} \mathrm{H}-\mathrm{NMR}\left(300 \mathrm{MHz}, d_{6}\right.$-DMSO): $\delta 1.21$ (s, 3H); $1.23(\mathrm{~s}, 3 \mathrm{H}), 3.08(\mathrm{~m}, 1 \mathrm{H}) ; 3.51$ (s, 3H); 7.48 (sharp m, 5H). ${ }^{13} \mathrm{C}-\mathrm{NMR}$ (75 MHz, $d_{6}$-DMSO): $\delta 20.6,31.8,52.4,110.2$, 127.6, 128.5, 130.6, 135.6, 157.5, 165.2, 167.2, 173.4. IR (KBr) $v_{\max } 1104,1282,1434,1603,1657,1725,3009,3406$ $\mathrm{cm}^{-1}$. ESIMS m/z $273\left(\left[\mathrm{M}+\mathrm{H}^{+}\right], \mathrm{C}_{15} \mathrm{H}_{16} \mathrm{~N}_{2} \mathrm{O}_{3} \text { requires 272). HRMS [M+H] }\right]^{+}: 273.1241$ (calc, Exact mass.: 273.1239). Analytical calculated for $\mathrm{C}_{15} \mathrm{H}_{16} \mathrm{~N}_{2} \mathrm{O}_{3}$ : C, 66.16; H, 5.92; N, 10.28. Found: C, 65.25, H, 5.64; N, 10.35 .

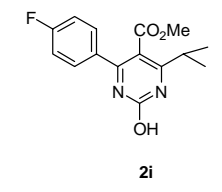

6-(4'-Fluoro)phenyl-2-hydroxy-4-isopropyl-5-methoxycarbonylpyrimidine (2i). ${ }^{1}{ }^{1} \mathrm{H}-\mathrm{NMR}$ (300 MHz, $d_{6}$-DMSO): $\delta 1.22$ $(\mathrm{d}, 6 \mathrm{H}, J=6.6) ; 3.08(\mathrm{~m}, 1 \mathrm{H}) ; 3.54(\mathrm{~s}, 3 \mathrm{H}) ; 7.32(\mathrm{dd}, 2 \mathrm{H}, J=8.7,8.9) ; 7.54(\mathrm{dd}, 2 \mathrm{H}, J=5.5,8.7) ; 12.3(\mathrm{br} \mathrm{s}, 1 \mathrm{H}) .{ }^{13} \mathrm{C}-$ NMR (75 MHz, $d_{6}$-DMSO): $\delta 20.5,31.8,52.5,110.3,115.36(\mathrm{~d}, J=22.0), 130.2(\mathrm{~d}, J=8.8), 132.5,157.4,163.4(\mathrm{~d}, J=$ 248.7), 167.1. IR (KBr) $v_{\max } 1203,1496,1614,1686,3148,3380 \mathrm{~cm}^{-1}$. ESIMS m/z $291\left(\left[\mathrm{M}+\mathrm{H}^{+}\right], \mathrm{C}_{15} \mathrm{H}_{15} \mathrm{FN}_{2} \mathrm{O}_{3}\right.$ requires 290). Analytical calculated for $\mathrm{C}_{15} \mathrm{H}_{15} \mathrm{FN}_{2} \mathrm{O}_{3}: \mathrm{C}, 62.06 ; \mathrm{H}, 5.20 ; \mathrm{N}, 9.65$. Found: C, 62.07, $\mathrm{H}, 5.14 ; \mathrm{N}, 9.57$. HRMS [M+H] $]^{+}: 291.11451$ (calc, Exact mass.: 291.11458).

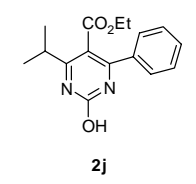

5-Ethoxycarbonyl-2-hydroxy-4-isopropyl-6-phenylpyrimidine (2j). ${ }^{1} \mathrm{H}-\mathrm{NMR}\left(300 \mathrm{MHz}, \mathrm{CDCl}_{3}\right): \delta 0.84(\mathrm{t}, 3 \mathrm{H}, J=7.2)$; 1.34 (s, 3H); 1.36 (s, 3H); 3.96 (q, 2H, $J=6.9) ; 7.34(\mathrm{~m}, 3 \mathrm{H}) ; 7.51(\mathrm{~m}, 2 \mathrm{H}) ; 13.1$ (br s, 1H). IR (KBr) $v_{\max } 1268,1438$, 1545, 1597, 1660, $1726 \mathrm{~cm}^{-1}$. ESIMS m/z $287\left(\left[\mathrm{M}+\mathrm{H}^{+}\right], \mathrm{C}_{16} \mathrm{H}_{18} \mathrm{~N}_{2} \mathrm{O}_{3}\right.$ requires 286). Analytical calculated for $\mathrm{C}_{16} \mathrm{H}_{17} \mathrm{~N}_{2} \mathrm{O}_{3}$ : C, 67.12; H, 6.34; N, 9.78. Found: C, 66.83, H, 6.34; N, 9.51. 


\section{General procedure for the base-catalyzed Biginelli-like three-component-coupling.}

Compounds $\mathbf{3 b - 3 \mathbf { j }}$ were prepared according to the literature procedure. A typical procedure is as follows: A solution of ethylacetoacetate $(483.8 \mathrm{mg}, 3.48 \mathrm{mmol})$, 2-(methylthio)pseudourea hydrogen sulfate (484 $\mathrm{mg}, 3.48 \mathrm{mmol})$ and acetoaldehyde $(0.128 \mathrm{~g}, 2.90 \mathrm{mmol})$ in $2.92 \mathrm{~mL}$ of dry DMF under nitrogen at room temperature was treated with sodium bicarbonate $(975 \mathrm{mg}, 11.6 \mathrm{mmol})$ and heated at $75^{\circ} \mathrm{C}$ for $3-4 \mathrm{~h}$. The reaction mixture was cooled to rt, then transferred into water $(8 \mathrm{ml})$ with ice-bath cooling over $\sim 1 \mathrm{~h}$, during which time the product solidified out from the solution. Stirring was continued at $\mathrm{rt}$ for $>3 \mathrm{~h}$, and the product was filtered. The cake was washed with water $(10 \mathrm{ml})$ and dried under 27 torr at $60{ }^{\circ} \mathrm{C}$ until constant weight was obtained. $654 \mathrm{mg}(2.25 \mathrm{mmol}, 78 \%)$ of $\mathbf{3 g}$ was obtained as pale-yellow solid.

Purification was accomplished by chromatography after the standard extractive workup with EtOAc when the product is not sufficiently crystalline.

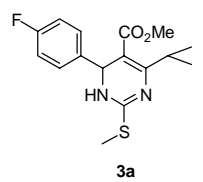

6-(4'-Fluorophenyl)-4-isopropyl-5-methoxycarbonyl-2-(methylthio)-1,6-dihydropyrimidine (3a). ${ }^{6}$ The title compound was prepared following the literature procedure from known S-1. Due to difficulty in isolation, the crude 3a was used without rigorous purification. The yield given in table 3. ESIMS m/z $323.2\left(\left[\mathrm{M}+\mathrm{H}^{+}\right], \mathrm{C}_{16} \mathrm{H}_{19} \mathrm{FN}_{2} \mathrm{O}_{2} \mathrm{~S}\right.$ requires 322$)$. HRMS $[\mathrm{M}+\mathrm{H}]^{+}: 323.1234$ (calc, Exact mass.: 323.1230).

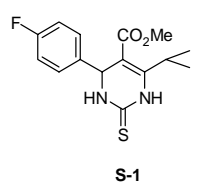

4-(4-Fluorophenyl)-6-isopropyl-5-methoxycarbonyl-1,2,3,4-tetrahydropyrimidine-2(1H)-thione $\quad(\mathbf{S}-\mathbf{1}){ }^{6} \quad$ The $\quad$ title compound was prepared following the literature procedure.

${ }^{1} \mathrm{H}-\mathrm{NMR}\left(300 \mathrm{MHz}, \mathrm{CDCl}_{3}\right): \delta 1.18(\mathrm{~d}, 3 \mathrm{H}, J=7.3) ; 1.23(\mathrm{~d}, 1 \mathrm{H}, J=7.5) ; 3.61(\mathrm{~s}, 3 \mathrm{H}) ; 4.12(\mathrm{~m}, 1 \mathrm{H}) ; 5.38(\mathrm{~d}, 1 \mathrm{H}, J=$ 3.0); $6.98(\mathrm{dd}, 2 \mathrm{H}, J=8.6,8.7) ; 7.22(\mathrm{dd}, 2 \mathrm{H}, J=5.1,8.6) .{ }^{13} \mathrm{C}-\mathrm{NMR}\left(75 \mathrm{MHz}, \mathrm{CDCl}_{3}\right): \delta 19.6,19.9,27.2,51.5,55.3$, 101.2, 115.9 (d, $J=21.4), 128.4(\mathrm{~d}, J=8.3), 138.1,151.0,161.5(\mathrm{~d}, J=247.6), 165.3,174.7 .{ }^{19} \mathrm{~F}-\mathrm{NMR}(282 \mathrm{MHz}$, $\left.\mathrm{CDCl}_{3}\right): \delta-113.7$. ESIMS m/z $309.1\left(\left[\mathrm{M}+\mathrm{H}^{+}\right], \mathrm{C}_{15} \mathrm{H}_{17} \mathrm{FN}_{2} \mathrm{O}_{2} \mathrm{~S}\right.$ requires 308).

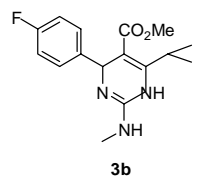

4-(4'-Fluorophenyl)-6-isopropyl-5-methoxycarbonyl-2-(methylamino)-1,4-dihydropyrimidine (3b). Yield: 77\%. This compound contained $\sim 20 \%$ impurity. A portion of the sample was chromatographically separated from the impurity for

6 End, N.; Richter, Y. . PCT WO 2004103977 A2 20041202 CAN 142:23301 AN 2004:1037079. 
analytical purpose. The yield for the oxidativive dehydrogenation for this substrate was corrected for the recovered impurity. ${ }^{1} \mathrm{H}-\mathrm{NMR}\left(300 \mathrm{MHz}, d_{6}\right.$-DMSO): $\delta 1.02$ (d, 3H, $J=6.6$ ); 1.09 (d, 1H, $\left.J=6.8\right) ; 2.70$ (s, 3H); 3.45 (s, 3H); 4.00 $(\mathrm{m}, 1 \mathrm{H}) ; 5.19(\mathrm{~s}, 1 \mathrm{H}) ; 6.29(\mathrm{br} \mathrm{s}, 1 \mathrm{H}) ; 7.08(\mathrm{dd}, 2 \mathrm{H}, J=8.8,8.9) ; 7.24(\mathrm{dd}, 2 \mathrm{H}, J=5.9,8.5) .{ }^{13} \mathrm{C}-\mathrm{NMR}\left(75 \mathrm{MHz}, d_{\sigma^{-}}\right.$ DMSO): $\delta 20.8,21.3,29.8,50.0,51.9,95.7,114.9(\mathrm{~d}, J=20.9), 128.0(\mathrm{~d}, J=7.7), 142.7(\mathrm{~d}, J=2.8), 154.9,161.2(\mathrm{~d}, J=$ 242.6), 166.3, 168.9. ${ }^{19}$ F-NMR (282 MHz, $d_{6}$-DMSO): $\delta-116.5$. IR (KBr) $v_{\max } 1227,1502,1649,1695,3299,3400 \mathrm{~cm}^{-1}$. ESIMS m/z $306.3\left(\left[\mathrm{M}+\mathrm{H}^{+}\right], \mathrm{C}_{16} \mathrm{H}_{20} \mathrm{FN}_{3} \mathrm{O}_{2} \text { requires 305). HRMS [M+H] }\right]^{+}: 306.1615$ (calc, Exact mass.: 306.1618 ).

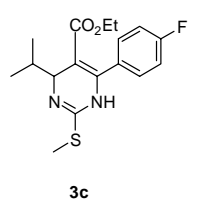

5-Ethoxycarbonyl-6-(4'-fluorophenyl)-4-isopropyl-2-(methylthio)-1,4-dihydropyrimidine (3c). ${ }^{7}$ The compound was purified by flash chromatography (0-40\% EtOAc/hexanes). Yield: $30 \%$. The title compound was reported but was never isolated. ${ }^{1} \mathrm{H}-\mathrm{NMR}\left(300 \mathrm{MHz}, \mathrm{CDCl}_{3}\right): \delta 1.08(\mathrm{t}, 3 \mathrm{H}, J=7.2) ; 1.30(\mathrm{~d}, 3 \mathrm{H}, J=6.8) ; 2.60(\mathrm{~s}, 3 \mathrm{H}) ; 3.16(\mathrm{~m}, 1 \mathrm{H}) ; 4.16(\mathrm{q}$, $2 \mathrm{H}, J=7.1) ; 7.11(\mathrm{dd}, 2 \mathrm{H}, J=8.7,8.7) ; 7.64(\mathrm{dd}, 2 \mathrm{H}, J=5.3,8.9) .{ }^{13} \mathrm{C}-\mathrm{NMR}\left(75 \mathrm{MHz}, \mathrm{CDCl}_{3}\right): \delta 13.7,14.2,21.6,33.2$, $61.8,115.2$ (d, $J=21.4),, 120.2,130.4(\mathrm{~d}, J=8.8), 133.9,165.3(\mathrm{~d}, J=250.3), 165.5,172.6,173.1 .{ }^{19} \mathrm{~F}-\mathrm{NMR}(282 \mathrm{MHz}$, $\left.\mathrm{CDCl}_{3}\right): \delta-111.1$. ESIMS m/z $337\left(\left[\mathrm{M}+\mathrm{H}^{+}\right], \mathrm{C}_{17} \mathrm{H}_{21} \mathrm{FN}_{2} \mathrm{O}_{3} \mathrm{~S}\right.$ requires 336).

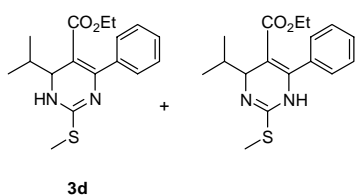

5-Ethoxycarbonyl-6-isopropyl-2-(methylamino)-4-phenyl-1,6-dihydropyrimidine (3d). The compound was purified by flask chromatography (0-30\% EtOAc/hexanes) and obtained as $\sim 1: 1$ mixture of regioisomers. Yield: 50\%. The mixture was used without further purification. ${ }^{1} \mathrm{H}-\mathrm{NMR}\left(300 \mathrm{MHz}, \mathrm{CDCl}_{3}\right)$ : $\delta$ 0.89-0.96 (m, $\left.12 \mathrm{H}\right) ; 1.91$ (br s, $\left.2 \mathrm{H}\right) ; 2.45$ (s, 6H); 3.80 (q, 4H, $J=7.0) ; 4.26$ (br s, 1H); 4.61 (br s, 1H); 5.88 (br s, 1H); 6.36 (br s, 1H); 7.33 (br s, 10H). IR (NaCl) $v_{\max }$ 1236, 1464, 1654, 1683, $3293 \mathrm{~cm}^{-1}$. ESIMS m/z $319\left(\left[\mathrm{M}+\mathrm{H}^{+}\right], \mathrm{C}_{17} \mathrm{H}_{22} \mathrm{~N}_{2} \mathrm{O}_{2} \mathrm{~S} \text { requires 318). HRMS [M+H] }\right]^{+}: 319.1487$ (calc, Exact mass.: 319.1480).

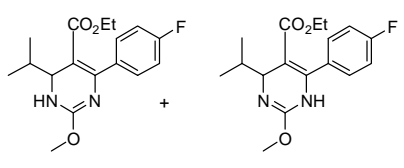

$3 e$

5-Ethoxycarbonyl-4-(4'-fluorophenyl)-6-isopropyl-2-(methoxy)-1,6-dihydropyrimidine (3e). Yield: 95\% ( 1:3.4 mixture of isomers). Major isomer. ${ }^{1} \mathrm{H}-\mathrm{NMR}\left(300 \mathrm{MHz}, \mathrm{CDCl}_{3}\right): \delta 0.96(\mathrm{~m}, 9 \mathrm{H}) ; 1.94(\mathrm{~m}, 1 \mathrm{H}) ; 3.84(\mathrm{~s}, 3 \mathrm{H}) ; 3.94(\mathrm{q}, 2 \mathrm{H}, J=$ 7.1); $4.36(\mathrm{dd}, 1 \mathrm{H}, J=3.3,3.6) ; 5.30($ br s, $1 \mathrm{H}) ; 7.01(\mathrm{dd}, 2 \mathrm{H}, J=8.6,8.9) ; 7.40(\mathrm{dd}, 2 \mathrm{H}, J=5.7,8.7) .{ }^{19} \mathrm{~F}-\mathrm{NMR}(282$ 
$\left.\mathrm{MHz}, \mathrm{CDCl}_{3}\right): \delta-114.4$. Minor isomer. ${ }^{1} \mathrm{H}-\mathrm{NMR}\left(300 \mathrm{MHz}, \mathrm{CDCl}_{3}\right): \delta 0.99(\mathrm{~m}, 6 \mathrm{H}) ; 1.02(\mathrm{~d}, 3 \mathrm{H}, J=5.8) ; 1.84(\mathrm{~m}$, $1 \mathrm{H}) ; 3.79$ (s, $3 \mathrm{H}) ; 3.95(\mathrm{~m}, 2 \mathrm{H}) ; 4.55(\mathrm{~d}, 1 \mathrm{H}, J=4.3) ; 6.02(\mathrm{br} \mathrm{s}, 1 \mathrm{H}) ; 7.09$ (dd, 2H, $J=8.6,8.7) ; 7.31(\mathrm{dd}, 2 \mathrm{H}, J=5.2$, 8.4). ${ }^{19} \mathrm{~F}-\mathrm{NMR}\left(282 \mathrm{MHz}, \mathrm{CDCl}_{3}\right): \delta-112.2$.

IR (film) $v_{\max } \mathrm{cm}^{-1}$. ESIMS m/z $321\left(\left[\mathrm{M}+\mathrm{H}^{+}\right], \mathrm{C}_{17} \mathrm{H}_{21} \mathrm{FN}_{2} \mathrm{O}_{3}\right.$ requires 320). HRMS $[\mathrm{M}+\mathrm{H}]^{+}: 321.1621$ (calc, Exact mass.: 321.1614).

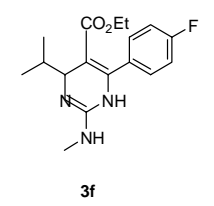

5-Ethoxycarbonyl-6-(4'-fluorophenyl)-4-isopropyl-2-(methylamino)-1,4-dihydropyrimidine (3f). ${ }^{8}$ Yield: $60 \%$. ${ }^{1} \mathrm{H}-\mathrm{NMR}$ (300 MHz, $\left.\mathrm{CDCl}_{3}\right): 0.92(\mathrm{~m}, 9 \mathrm{H}) ; 1.95(\mathrm{~m}, 1 \mathrm{H}) ; 2.24(\mathrm{~s}, 3 \mathrm{H}) ; 3.88(\mathrm{q}, 2 \mathrm{H}, J=7.2) ; 4.27(\mathrm{~d}, 1 \mathrm{H}, J=4.5) ; 7.01(\mathrm{dd}, 2 \mathrm{H}, J=$ 8.8, 8.7); $7.34(\mathrm{dd}, 2 \mathrm{H}, J=5.3,8.9) .{ }^{13} \mathrm{C}-\mathrm{NMR}\left(75 \mathrm{MHz}, \mathrm{CDCl}_{3}\right): \delta 13.9,16.5,18.5,26.6,34.6,55.9,59.1,97.1,114.3(\mathrm{~d}$, $J=21.4), 130.2(\mathrm{~d}, J=7.7), 138.2,155.9,162.6(\mathrm{~d}, J=246), 167.2 .{ }^{19} \mathrm{~F}-\mathrm{NMR}\left(282 \mathrm{MHz}, \mathrm{CDCl}_{3}\right): \delta-115.0$. ESIMS m/z $320\left(\left[\mathrm{M}+\mathrm{H}^{+}\right], \mathrm{C}_{17} \mathrm{H}_{22} \mathrm{FN}_{3} \mathrm{O}_{2}\right.$ requires 320$)$.

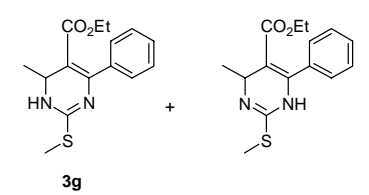

5-Ethoxycarbonyl-6-methyl-2-(methylthio)-4-phenyl-1,6-dihydropyrimidine $\quad(\mathbf{3 g}) . \quad$ Yield: $78 \% \quad(\sim 1: 1.5$ mixture of isomers).

${ }^{1} \mathrm{H}-\mathrm{NMR}\left(300 \mathrm{MHz}, \mathrm{CDCl}_{3}\right): 0.93(\mathrm{~m}$, major $3 \mathrm{H}+\operatorname{minor} 3 \mathrm{H}) ; 1.29(\mathrm{~d}$, minor $3 \mathrm{H}, J=6.0) ; 1.30(\mathrm{~d}$, major 3H, $J=6.6)$; 2.47 (s, major 3H); 2.49 (s, minor 3H); $3.93(\mathrm{~m}$, major $2 \mathrm{H}+\operatorname{minor} 2 \mathrm{H}) ; 4.52(\mathrm{~m}$, minor $1 \mathrm{H}) ; 4.77$ (q, major 1H, $J=6.6$ ); 5.61 (br s, minor 1H); 6.22 (br s, major $1 \mathrm{H}) ; 7.32$ (m, major $2 \mathrm{H}+\operatorname{minor} 2 \mathrm{H}) ; 7.40$ (m, major $2 \mathrm{H}+\operatorname{minor} 2 \mathrm{H})$. IR $(\mathrm{KBr})$ $v_{\max } 1132,1203,1498,1624,16763050,3170 \mathrm{~cm}^{-1}$. ESIMS m/z $291\left(\left[\mathrm{M}+\mathrm{H}^{+}\right], \mathrm{C}_{15} \mathrm{H}_{18} \mathrm{~N}_{2} \mathrm{O}_{2} \mathrm{~S}\right.$ requires 290). Analytical calculated for $\mathrm{C}_{15} \mathrm{H}_{18} \mathrm{~N}_{2} \mathrm{O}_{2} \mathrm{~S}$ : C, 62.04; H, 6.24; N, 9.64. Found: C, 61.84, H, 6.27; N, 9.54.
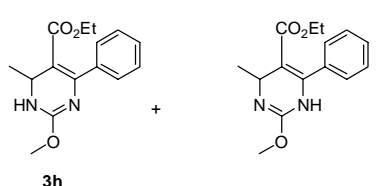

5-Ethoxycarbonyl-6-methyl-2-methoxy-4-phenyl -1,6-dihydropyrimidine (3h). Yield: 60\% (obtained as $\sim 1: 1$ mixture of isomers). ${ }^{1} \mathrm{H}-\mathrm{NMR}\left(300 \mathrm{MHz}, \mathrm{CDCl}_{3}\right): \delta 0.89(\mathrm{~m}, 6 \mathrm{H}) ; 1.29(\mathrm{~m}, 6 \mathrm{H}) ; 3.73(\mathrm{~s}, 3 \mathrm{H}) ; 3.82(\mathrm{~s}, 3 \mathrm{H}) ; 3.92(\mathrm{~m}, 4 \mathrm{H}) ; 4.52$ $(\mathrm{m}, 1 \mathrm{H}) ; 4.65$ (q, 1H, J = 6.6); 5.34 (br s, 1H); 6.12 (br s, 1H); 7.29 (m, 4H); 7.37 (m, 4H). IR (KBr) $v_{\max } 1038,1246$, 1447, 1503, 1601, 1682, 3054, $3121 \mathrm{~cm}^{-1}$. ESIMS m/z $275.3\left(\left[\mathrm{M}+\mathrm{H}^{+}\right], \mathrm{C}_{15} \mathrm{H}_{18} \mathrm{~N}_{2} \mathrm{O}_{3} \text { requires 274). HRMS [M+H }\right]^{+}$: 275.1401 (calc, Exact mass.: 275.1396).

\footnotetext{
7 Hirai, K.; Ishiba, T.; Koike, H.; Watanabe, M. Eur. Pat. Appl. 1993, 18pp. EP 521471 A1 19930107 CAN 118:254949 AN 1993:254949.

8 Lee, N.; Bailey, M. PCT Int. Appl. 2004, 19pp. WO 20044054986 A2 20040701 CAN 141:71558 AN 2004:534182.
} 


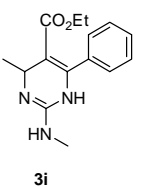

5-Methoxycarbonyl-2-methylamino-4-methyl-6-phenyl-1,4-dihydropyrimidine (3i). Yield: 97\%. ${ }^{1} \mathrm{H}-\mathrm{NMR}(300 \mathrm{MHz}$, $\left.\mathrm{CDCl}_{3}\right): \delta 0.84(\mathrm{t}, 3 \mathrm{H}, J=7.2) ; 1.28(\mathrm{~d}, 3 \mathrm{H}, J=5.8) ; 2.11(\mathrm{~s}, 3 \mathrm{H}) ; 3.84(\mathrm{q}, 2 \mathrm{H}, J=7.2) ; 4.52(\mathrm{q}, 1 \mathrm{H}, J=6.2) ; 7.30(\mathrm{~m}$, 5H). ${ }^{13} \mathrm{C}-\mathrm{NMR}\left(75 \mathrm{MHz}, \mathrm{CDCl}_{3}\right): \delta 13.8,23.7,26.1,46.5,58.9,99.2,127.5,128.1,142.6,155.3,160.0,167.0 . \mathrm{IR}(\mathrm{KBr})$ $v_{\max } 1067,1227,1375,1588,1644,3236,3331 \mathrm{~cm}^{-1}$. ESIMS m/z $274.3\left(\left[\mathrm{M}+\mathrm{H}^{+}\right], \mathrm{C}_{15} \mathrm{H}_{19} \mathrm{~N}_{3} \mathrm{O}_{2}\right.$ requires 273). HRMS $[\mathrm{M}+\mathrm{H}]^{+}: 274.1563$ (calc, Exact mass.: 274.1556).

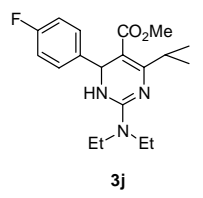

6-(4'-Fluorophenyl)-4-isopropyl-5-methoxycarbonyl-2-diethylamino-1,6-dihydropyrimidine $\quad$ (3j). $\quad$ Yield: $\quad$ 75\% (contaminated with $\sim 2 \%$ impurity). ${ }^{1} \mathrm{H}-\mathrm{NMR}\left(300 \mathrm{MHz}, \mathrm{CDCl}_{3}\right): \delta 1.08(\mathrm{~m}, 3 \mathrm{H}) ; 1.10(\mathrm{~d}, 3 \mathrm{H}, J=7.0) ; 1.16(\mathrm{~d}, 3 \mathrm{H}, J=$ 6.6); 3.27 (m, 2H); $3.44(\mathrm{~m}, 2 \mathrm{H}) ; 4.00(\mathrm{~m}, 1 \mathrm{H}) ; 4.96$ (br s, $1 \mathrm{H}) ; 5.34$ (d, $1 \mathrm{H}, J=3.0) ; 6.93(\mathrm{dd}, 2 \mathrm{H}, J=8.7,9.2) ; 7.25(\mathrm{~m}$, 2H). ${ }^{13} \mathrm{C}-\mathrm{NMR}\left(75 \mathrm{MHz}, \mathrm{CDCl}_{3}\right): \delta 13.8,20.9,21.4,30.0,41.4,50.0,51.2,94.4,114.9(\mathrm{~d}, J=21.4), 127.9(\mathrm{~d}, J=7.7)$, $142.4(\mathrm{~d}, J=3.3), 153.2,161.1(\mathrm{~d}, J=242), 166.2,169.3 .{ }^{19} \mathrm{~F}-\mathrm{NMR}\left(282 \mathrm{MHz}, \mathrm{CDCl}_{3}\right): \delta-116.5 . \mathrm{IR}(\mathrm{KBr}) v_{\max } 1186$, 1227, 1351, 1576, 1641, $3336 \mathrm{~cm}^{-1}$. ESIMS m/z $348\left(\left[\mathrm{M}+\mathrm{H}^{+}\right], \mathrm{C}_{19} \mathrm{H}_{26} \mathrm{FN}_{3} \mathrm{O}_{2}\right.$ requires 347). Analytical calculated for $\mathrm{C}_{19} \mathrm{H}_{26} \mathrm{~N}_{3} \mathrm{O}_{2}:$ C, 65.68; H, 7.54; N, 12.09. Found: C, 65.93, H, 7.81; N, 12.20.

Pyrimidines $\mathbf{4 a - 4 j}$, 5c-5f were obtained following the general procedure for oxidative dehydrogenation described above for compounds $\mathbf{1 a - 1 j}$. In cases where products are not crystalline, purification was performed by flash chromatography after standard extractive workup with EtOAc.

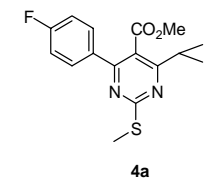

6-(4'-Fluorophenyl)-4-isopropyl-5-methoxycarbonyl-2-(methylthio)pyrimidine (4a). ${ }^{6,9}{ }^{1} \mathrm{H}-\mathrm{NMR}\left(300 \mathrm{MHz}, \mathrm{CDCl}_{3}\right): \delta$ $1.31(\mathrm{~d}, 3 \mathrm{H}, J=6.8) ; 2.62(\mathrm{~s}, 3 \mathrm{H}) ; 3.13(\mathrm{~m}, 1 \mathrm{H}) ; 3.70(\mathrm{~s}, 3 \mathrm{H}) ; 7.13(\mathrm{dd}, 2 \mathrm{H}, J=8.6,8.6) ; 7.65(\mathrm{dd}, 2 \mathrm{H}, J=5.4,8.9) .{ }^{13} \mathrm{C}-$ NMR (75 MHz, $\left.\mathrm{CDCl}_{3}\right): \delta 14.2,21.7,33.4,52.6,115.7(\mathrm{~d}, J=21.4), 119.9,130.3(\mathrm{~d}, J=8.8), 133.8,163.9(\mathrm{~d}, J=253.6)$, 168.9, 172.8, 173.2. ${ }^{19} \mathrm{~F}-\mathrm{NMR}\left(282 \mathrm{MHz}, \mathrm{CDCl}_{3}\right): \delta-110.8$. ESIMS m/z $321\left(\left[\mathrm{M}+\mathrm{H}^{+}\right], \mathrm{C}_{16} \mathrm{H}_{17} \mathrm{FN}_{2} \mathrm{O}_{2} \mathrm{~S}\right.$ requires 320$)$.

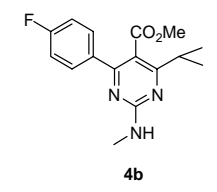

\footnotetext{
9 Ahmad, S.; Robl, J. A.; Ngu, K. PCT Int Appl. 2005, 103pp. WO 2005030758 A1 20050407 CAN 142:373859 AN 2005:300435.
} 
6-(4'-Fluorophenyl)-4-isopropyl-5-methoxycarbonyl-2-(methylamino)pyrimidine (4b). ${ }^{1,} 6$ The starting material $\mathbf{3 b}$ contained $\sim 20 \%$ of an impurity. The yield was corrected based on recovered impurity after the chromatographic separation of the product. ${ }^{1} \mathrm{H}-\mathrm{NMR}\left(300 \mathrm{MHz}, \mathrm{CDCl}_{3}\right): \delta 1.24(\mathrm{~d}, 6 \mathrm{H}, J=6.8) ; 2.78$ (br s, 3H); $3.18(\mathrm{~m}, 1 \mathrm{H}) ; 3.57(\mathrm{~s}$, $3 \mathrm{H}) ; 7.07(\mathrm{dd}, 2 \mathrm{H}, J=8.6,8.7) ; 7.56(\mathrm{br} \mathrm{m}, 2 \mathrm{H}) .{ }^{13} \mathrm{C}-\mathrm{NMR}\left(75 \mathrm{MHz}, \mathrm{CDCl}_{3}\right): \delta 21.5,27.8,32.7,51.9,113.9,115.2(\mathrm{~d}, J$ $=21.4), 129.8(\mathrm{~d}, J=8.3), 135.2,162.1,163.4(\mathrm{~d}, J=249.2), 164.1,169.6,174.8 .{ }^{19} \mathrm{~F}-\mathrm{NMR}\left(282 \mathrm{MHz}, \mathrm{CDCl}_{3}\right): \delta-112.0$. $\mathrm{IR}(\mathrm{NaCl}) v_{\max } 1159,1263,1402,1599,1721,3272,3408 \mathrm{~cm}^{-1}$. ESIMS m/z $304\left(\left[\mathrm{M}+\mathrm{H}^{+}\right], \mathrm{C}_{16} \mathrm{H}_{18} \mathrm{FN}_{3} \mathrm{O}_{2}\right.$ requires 303).

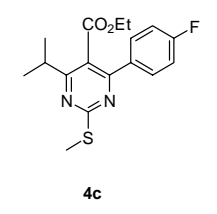

5-Ethoxycarbonyl-6-(4'-fluorophenyl)-4-isopropyl-2-(methylthio)pyrimidine (4c). ${ }^{8,}{ }^{1}{ }^{1} \mathrm{H}-\mathrm{NMR}\left(300 \mathrm{MHz}, \mathrm{CDCl}_{3}\right): \delta$ $1.08(\mathrm{t}, 3 \mathrm{H}, J=7.2), 1.30(\mathrm{~d}, 6 \mathrm{H}, J=6.8) ; 2.60(\mathrm{~s}, 3 \mathrm{H}) ; 3.16(\mathrm{~m}, 1 \mathrm{H}) ; 4.16(\mathrm{q}, 2 \mathrm{H}, J=7.2) ; 7.11(\mathrm{dd}, 2 \mathrm{H}, J=8.7,8.7)$; $7.64(\mathrm{dd}, 2 \mathrm{H}, J=5.5,8.9) .{ }^{13} \mathrm{C}-\mathrm{NMR}\left(75 \mathrm{MHz}, \mathrm{CDCl}_{3}\right): \delta 13.7,14.2 .21 .7,33.3,61.8,115.6(\mathrm{~d}, J=22.0), 120.2,130.4(\mathrm{~d}$, $J=8.2), 134.0(\mathrm{~d}, J=3.3), 162.4,163.9(\mathrm{~d}, J=250.8), 168.3,172.7,173.1 .{ }^{19} \mathrm{~F}-\mathrm{NMR}\left(282 \mathrm{MHz}, \mathrm{CDCl}_{3}\right): \delta-111.0$. ESIMS m/z $335\left(\left[\mathrm{M}+\mathrm{H}^{+}\right], \mathrm{C}_{17} \mathrm{H}_{19} \mathrm{FN}_{2} \mathrm{O}_{2} \mathrm{~S}\right.$ requires 334$)$.

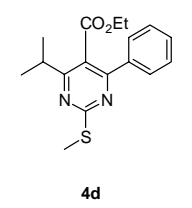

5-Ethoxycarbonyl-6-(4'-fluorophenyl)-4-isopropyl-2-(methylamino)pyrimidine (4d). ${ }^{1} \mathrm{H}-\mathrm{NMR}\left(300 \mathrm{MHz}, \mathrm{CDCl}_{3}\right): \delta$ $1.03(\mathrm{t}, 3 \mathrm{H}, J=7.1) ; 2.62(\mathrm{~s}, 3 \mathrm{H}) ; 3.20(\mathrm{~m}, 1 \mathrm{H}) ; 4.15(\mathrm{q}, 2 \mathrm{H}, J=7.2) ; 7.44(\mathrm{~m}, 3 \mathrm{H}) ; 7.64(\mathrm{~m}, 2 \mathrm{H}) .{ }^{13} \mathrm{C}-\mathrm{NMR}(75 \mathrm{MHz}$ $\left.\mathrm{CDCl}_{3}\right): \delta 13.6,14.2,33.2,61.7,120.4,128.3,128.4,129.9,137.9,163.6,168.3,172.6,173.0 . \quad \mathrm{IR}(\mathrm{NaCl}) v_{\max } 1219,1529$, $1275 \mathrm{~cm}^{-1}$. ESIMS m/z $317.2\left(\left[\mathrm{M}+\mathrm{H}^{+}\right], \mathrm{C}_{17} \mathrm{H}_{20} \mathrm{~N}_{2} \mathrm{O}_{2} \mathrm{~S}\right.$ requires 316). HRMS $[\mathrm{M}+\mathrm{H}]^{+}: 317.1316$ (calc, Exact mass.: 317.1324).

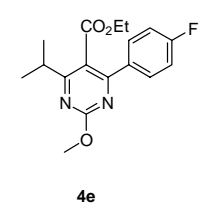

5-Ethoxycarbonyl-6-(4'-fluorophenyl)-4-isopropyl-2-methoxypyrimidine (4e). ${ }^{1} \mathrm{H}-\mathrm{NMR}\left(300 \mathrm{MHz}, \mathrm{CDCl}_{3}\right): \delta 1.06(\mathrm{t}$, $3 \mathrm{H}, J=7.2) ; 1.28(\mathrm{~d}, 6 \mathrm{H}, J=6.8) ; 3.17(\mathrm{~m}, 1 \mathrm{H}) ; 4.04(\mathrm{~s}, 3 \mathrm{H}) ; 4.14(\mathrm{q}, 2 \mathrm{H}, J=7.1) ; 7.09$ (dd, $2 \mathrm{H}, J=8.5,8.8) ; 7.64(\mathrm{dd}$, $2 \mathrm{H}, J=5.3,8.8) .{ }^{13} \mathrm{C}-\mathrm{NMR}\left(75 \mathrm{MHz}, \mathrm{CDCl}_{3}\right): \delta 13.6,21.5,33.0,54.9,61.7,115.5(\mathrm{~d}, J=22.0), 119.1,130.3(\mathrm{~d}, J=3.3)$, 133.9, $163.8(\mathrm{~d}, J=250.3), 164.8,165.0,168.4,176.4 .{ }^{19} \mathrm{~F}-\mathrm{NMR}\left(282 \mathrm{MHz}, \mathrm{CDCl}_{3}\right): \delta-111.0 . \operatorname{IR}(\mathrm{KBr}) v_{\max } 1289$, 1467, 1600, $1700 \mathrm{~cm}^{-1}$. ESIMS m/z $319.3\left(\left[\mathrm{M}+\mathrm{H}^{+}\right], \mathrm{C}_{17} \mathrm{H}_{19} \mathrm{FN}_{2} \mathrm{O}_{3}\right.$ requires 318). HRMS $[\mathrm{M}+\mathrm{H}]^{+}: 319.1454$ (calc, Exact mass.: 319.1458).

${ }_{10}$ Watanabe, M.; Koike, H.; Ishiba, T.; Okada, T.; Seo, S.; Hirai, K. Bioorg. Med. Chem. 1997, 5, 437. 


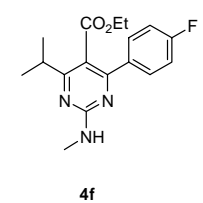

5-Ethoxycarbonyl-6-(4'-fluorophenyl)-4-isopropyl-2-(methylamino)pyrimidine (4f). ${ }^{1,6}{ }^{1} \mathrm{H}-\mathrm{NMR}\left(300 \mathrm{MHz} \mathrm{CDCl}_{3}\right): \delta$ $1.00(\mathrm{t}, 3 \mathrm{H}, J=7.1), 1.25(\mathrm{~d}, 6 \mathrm{H}, J=6.6), 2.98(\mathrm{~d}, 1 \mathrm{H}, J=4.9), 3.20(\mathrm{~m}, 1 \mathrm{H}) ; 4.06(\mathrm{q}, 2 \mathrm{H}, J=7.2) ; 5.53(\mathrm{br} \mathrm{s}, 1 \mathrm{H}) ; 7.08$ $(\mathrm{dd}, 2 \mathrm{H}, J=8.6,8.7) ; 7.56(\mathrm{~m}, 2 \mathrm{H}) .{ }^{13} \mathrm{C}-\mathrm{NMR}\left(75 \mathrm{MHz}, \mathrm{CDCl}_{3}\right): \delta 13.6,21.6,28.1,32.6,61.1,114.5,115.3(\mathrm{~d}, J=22.0)$, $129.9(\mathrm{~d}, J=8.2), 135.4,162.1,163.5(\mathrm{~d}, J=249.2), 164.3,169.1,174.8 .{ }^{19} \mathrm{~F}-\mathrm{NMR}\left(282 \mathrm{MHz}, \mathrm{CDCl}_{3}\right): \delta-113.0$. ESIMS m/z $318\left(\left[\mathrm{M}+\mathrm{H}^{+}\right], \mathrm{C}_{17} \mathrm{H}_{20} \mathrm{FN}_{3} \mathrm{O}_{2}\right.$ requires 317).

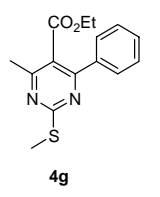

5-Ethoxycarbonyl-4-methyl-2-(methylthio)-6-phenylpyrimidine $(\mathbf{4 g}) .{ }^{1} \mathrm{H}-\mathrm{NMR}\left(300 \mathrm{MHz}, \mathrm{CDCl}_{3}\right): \delta 1.02(\mathrm{t}, 3 \mathrm{H}, J=$ 7.2); $2.55(\mathrm{~s}, 3 \mathrm{H}) ; 2.60(\mathrm{~s}, 3 \mathrm{H}) ; 4.14(\mathrm{q}, 2 \mathrm{H}, J=7.1) ; 7.43(\mathrm{~m}, 3 \mathrm{H}) ; 7.62(\mathrm{~m}, 2 \mathrm{H}) .{ }^{13} \mathrm{C}-\mathrm{NMR}\left(75 \mathrm{MHz}, \mathrm{CDCl}_{3}\right): \delta 13.5$, 14.1, 22.6, 61.6, 120.9, 128.3, 128.4, 130.0, 137.7, 163.5, 165.4, 168.1, 172.4. IR (NaCl) $v_{\max } 1081,1232,1535,1722$ $\mathrm{cm}^{-1}$. ESIMS m/z $289.2\left(\left[\mathrm{M}+\mathrm{H}^{+}\right], \mathrm{C}_{15} \mathrm{H}_{16} \mathrm{~N}_{2} \mathrm{O}_{2} \mathrm{~S} \text { requires 288). HRMS [M+H }\right]^{+}: 289.1003$ (calc, Exact mass.: 289.1011).

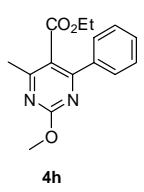

5-Ethoxycarbonyl-4-methyl-2-methoxy-6-phenylpyrimidine (4h). ${ }^{1} \mathrm{H}-\mathrm{NMR}\left(300 \mathrm{MHz}, \mathrm{CDCl}_{3}\right): \delta 1.01(\mathrm{t}, 3 \mathrm{H}, J=7.1)$; $2.56(\mathrm{~s}, 3 \mathrm{H}) ; 4.05(\mathrm{~s}, 3 \mathrm{H}), 4.13(\mathrm{q}, 2 \mathrm{H}, J=7.2) ; 7.43(\mathrm{~m}, 3 \mathrm{H}) ; 7.63(\mathrm{~m}, 2 \mathrm{H}) .{ }^{13} \mathrm{C}-\mathrm{NMR}\left(75 \mathrm{MHz}, \mathrm{CDCl}_{3}\right): \delta 13.6,22.7$, 55.0, 61.6, 119.9, 128.2, 128.3, 130.0, 137.8, 164.5, 166.4, 168.3, 168.7. IR (NaCl) $v_{\max } 1217,1447,1585,1725 \mathrm{~cm}^{-1}$. ESIMS m/z $273\left(\left[\mathrm{M}+\mathrm{H}^{+}\right], \mathrm{C}_{15} \mathrm{H}_{16} \mathrm{~N}_{2} \mathrm{O}_{3}\right.$ requires 272). HRMS $[\mathrm{M}+\mathrm{H}]^{+}: 273.1230$ (calc, Exact mass.: 273.1239).

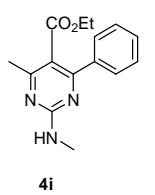

5-Methoxycarbonyl-2-methylamino-4-methyl-6-phenylpyrimidine (4i). ${ }^{1} \mathrm{H}-\mathrm{NMR}\left(300 \mathrm{MHz}, \mathrm{CDCl}_{3}\right): \delta 0.92(\mathrm{t}, 3 \mathrm{H}, J=$ 7.2); 2.49 (s, 3H); 2.93 (d, 3H, $J=4.8) ; 4.03$ (q, 2H, $J=7.2) ; 7.39$ (m, 3H); 7.54 (br s, 2H). ${ }^{13} \mathrm{C}-\mathrm{NMR}\left(75 \mathrm{MHz} \mathrm{CDCl}_{3}\right)$ : $\delta$ 13.5, 23.0, 28.1, 60.9, 114.9, 127.9, 128.2, 129.3, 139.2, 161.8, 166.1, 168.9. IR (NaCl) $v_{\max } 1266,1558,1595,1716$, $3272 \mathrm{~cm}^{-1}$. ESIMS m/z $272.3\left(\left[\mathrm{M}+\mathrm{H}^{+}\right], \mathrm{C}_{15} \mathrm{H}_{17} \mathrm{~N}_{3} \mathrm{O}_{2}\right.$ requires 271). HRMS $[\mathrm{M}+\mathrm{H}]^{+}: 272.1397$ (calc, Exact mass.: 272.1399).

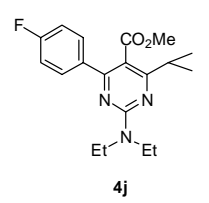


6-(4'-Fluorophenyl)-4-isopropyl-5-methoxycarbonyl-2-(diethylamino)pyrimidine (4j). ${ }^{1} \mathrm{H}-\mathrm{NMR}\left(300 \mathrm{MHz}, \mathrm{CDCl}_{3}\right): \delta$ 1.20 (t, 6H, $J=7.2) ; 1.25(\mathrm{~d}, 6 \mathrm{H}, J=6.8) ; 3.18(\mathrm{~m}, 1 \mathrm{H}) ; 3.59$ (s, 3H); 3.68 (q, 2H, $J=7.0) ; 7.08$ (dd, 2H, $J=8.7,8.8)$; $7.59(\mathrm{dd}, 2 \mathrm{H}, J=5.5,8.9) .{ }^{13} \mathrm{C}-\mathrm{NMR}\left(75 \mathrm{MHz}, \mathrm{CDCl}_{3}\right): \delta 13.2,21.7,32.7,42.2,51.9,112.2,115.2(\mathrm{~d}, J=21.4), 130.0(\mathrm{~d}$, $J=8.7), 135.9(\mathrm{~d}, J=3.3), 160.1,163.5(\mathrm{~d}, J=248.6), 163.8,170.3,174.3 .{ }^{19} \mathrm{~F}-\mathrm{NMR}\left(282 \mathrm{MHz}, \mathrm{CDCl}_{3}\right): \delta-112.6 . \quad$ IR $(\mathrm{NaCl}) v_{\max } 1198,1259,1560,1600,1720 \mathrm{~cm}^{-1}$. ESIMS m/z $346\left(\left[\mathrm{M}+\mathrm{H}^{+}\right], \mathrm{C}_{19} \mathrm{H}_{24} \mathrm{FN}_{3} \mathrm{O}_{2}\right.$ requires 345). HRMS $[\mathrm{M}+\mathrm{H}]^{+}: 346.1943$ (calc, Exact mass.: 346.1931).

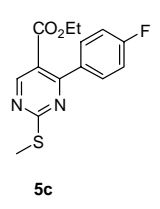

5-Ethoxycarbonyl-6-(4'-fluorophenyl)-2-(methylthio)pyrimidine (5c). ${ }^{1} \mathrm{H}-\mathrm{NMR}\left(300 \mathrm{MHz}, \mathrm{CDCl}_{3}\right): \delta 1.18(\mathrm{t}, 3 \mathrm{H}, J=$ 7.0); 2.63 (s, 3H); 4.24 (q, 2H, $J=7.1) ; 7.14(\mathrm{dd}, 2 \mathrm{H}, J=8.7,8.6) ; 7.61(\mathrm{dd}, 2 \mathrm{H}, J=5.3,8.9) .{ }^{13} \mathrm{C}-\mathrm{NMR}(75 \mathrm{MHz}$, $\left.\mathrm{CDCl}_{3}\right): \delta 13.9,14.4,61.7,115.3(\mathrm{~d}, J=22.0), 119.0,131.1(\mathrm{~d}, J=8.8), 133.5,159.0,164.1(\mathrm{~d}, J=251), 164.9,166.0$, 175.2. ${ }^{19}$ F-NMR (282 MHz, $\left.\mathrm{CDCl}_{3}\right): \delta-110.6$. IR $(\mathrm{NaCl}) v_{\max } 1204,1286,1557,1604,1728 \mathrm{~cm}^{-1}$. ESIMS m/z 293.2 $\left(\left[\mathrm{M}+\mathrm{H}^{+}\right], \mathrm{C}_{14} \mathrm{H}_{13} \mathrm{FN}_{2} \mathrm{O}_{2} \mathrm{~S}\right.$ requires 292). HRMS [M+H] ${ }^{+}: 293.0757$ (calc, Exact mass.: 293.0760).

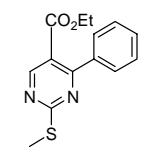

$5 d$

5-Ethoxycarbonyl-6-fluorophenyl-2-(methylamino)pyrimidine (5d). ${ }^{11}{ }^{1} \mathrm{H}-\mathrm{NMR}\left(300 \mathrm{MHz}, \mathrm{CDCl}_{3}\right): \delta 1.10(\mathrm{t}, 3 \mathrm{H}, J=$ 7.2); 2.62 (s, 3H); 4.19 (q, 2H, $J=7.2) ; 7.43(\mathrm{~m}, 3 \mathrm{H}) ; 7.58(\mathrm{~m}, 2 \mathrm{H}) ; 8.90(\mathrm{~s}, 1 \mathrm{H}) .{ }^{13} \mathrm{C}-\mathrm{NMR}\left(75 \mathrm{MHz}, \mathrm{CDCl}_{3}\right): \delta 13.7$, $14.3,61.5,119.3,12801,128.8,130.1,137.5,158.8,166.0,166.1,175.0 . \quad \mathrm{IR}(\mathrm{NaCl}) v_{\max } 1289,1401,1582,1601,1717$ $\mathrm{cm}^{-1}$. ESIMS m/z $275\left(\left[\mathrm{M}+\mathrm{H}^{+}\right], \mathrm{C}_{14} \mathrm{H}_{14} \mathrm{~N}_{2} \mathrm{O}_{2} \mathrm{~S}\right.$ requires 274).

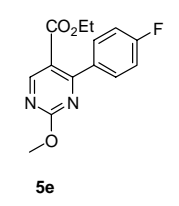

5-Ethoxycarbonyl-6-(4'-fluorophenyl)-2-methoxypyrimidine (5e). ${ }^{1} \mathrm{H}-\mathrm{NMR}\left(300 \mathrm{MHz}, \mathrm{CDCl}_{3}\right): \delta 1.19(\mathrm{t}, 3 \mathrm{H}, J=7.2)$; $4.11(\mathrm{~s}, 3 \mathrm{H}) ; 4.24$ (q, 2H, $J=7.2) ; 7.14(\mathrm{dd}, 2 \mathrm{H}, J=8.6,8.7) ; 7.62(\mathrm{dd}, 2 \mathrm{H}, J=5.3,8.7) .{ }^{19} \mathrm{~F}-\mathrm{NMR}\left(282 \mathrm{MHz}, \mathrm{CDCl}_{3}\right)$ : $\delta-110.6 \mathrm{IR}(\mathrm{NaCl}) v_{\max } 1243,1288,1474,1575,1720 \mathrm{~cm}^{-1}$. ESIMS m/z $277.2\left(\left[\mathrm{M}+\mathrm{H}^{+}\right], \mathrm{C}_{14} \mathrm{H}_{13} \mathrm{FN}_{2} \mathrm{O}_{3}\right.$ requires 276). HRMS [M+H] $]^{+} 277.0981$ (calc, Exact mass.: 277.0988).

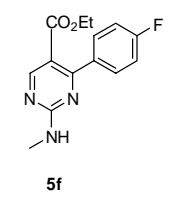

${ }^{11}$ Breaux, E. J.; Zwikelmaier, K. E. J. Heterocyc. Chem. 1981, 18, 183. 
5-Ethoxycarbonyl-6-(4'-fluorophenyl)-2-(methylamino)pyrimidine (5f). The compound obtained as $\sim 1: 2.5$ mixture of isomers. The structure of the minor isomer was not characterized. ${ }^{1} \mathrm{H}-\mathrm{NMR}\left(300 \mathrm{MHz}, \mathrm{CDCl}_{3}\right): \delta 1.13(\mathrm{t}$, major $3 \mathrm{H}+$ minor $3 \mathrm{H}, J=7.2)$; 3.00 (d, major $3 \mathrm{H}+\operatorname{minor} 3 \mathrm{H}, J=5.1$ ); 4.15 (q, major $2 \mathrm{H}+\operatorname{minor} 2 \mathrm{H}, J=7.2$ ); 5.86 (br s, minor $1 \mathrm{H}) ; 6.02$ (br s, major $1 \mathrm{H}) ; 7.09$ (dd, major $2 \mathrm{H}+\operatorname{minor} 2 \mathrm{H}, J=8.6,8.7$ ); 7.49 (br s, major $2 \mathrm{H}+$ minor $2 \mathrm{H}$ ); 8.81 (br s, minor $1 \mathrm{H}$ ); 8.90 (br s, $1 \mathrm{H}$ ). ${ }^{19} \mathrm{~F}-\mathrm{NMR}\left(282 \mathrm{MHz}, \mathrm{CDCl}_{3}\right.$ ): $\delta-112.1$ (major), -110.0 (minor IR $(\mathrm{NaCl}) v_{\max } 1158,1225$, $1508,1580,1603,1706,3270 \mathrm{~cm}^{-1}$. ESIMS m/z $276.3\left(\left[\mathrm{M}+\mathrm{H}^{+}\right], \mathrm{C}_{14} \mathrm{H}_{14} \mathrm{FN}_{3} \mathrm{O}_{2}\right.$ requires 275). HRMS $[\mathrm{M}+\mathrm{H}]^{+}: 276.1137$ (calc, Exact mass.: 276.1148).

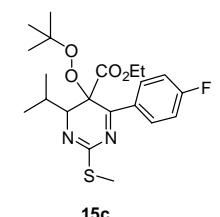

5-(tert-Butylperoxy)-5-ethoxycarbonyl-6-(4-fluorophenyl)-4-isopropyl-2-(methylthio)-4,5-dihydropyrimidine (15c). ${ }^{1} \mathrm{H}$ NMR (300 MHz, $\left.\mathrm{CDCl}_{3}\right): \delta 0.79(\mathrm{~d}, 3 \mathrm{H}, J=6.5) ; 1.05(\mathrm{~s}, 9 \mathrm{H}) ; 1.13(\mathrm{~m}, 5 \mathrm{H}) ; 1.91(\mathrm{~m}, 1 \mathrm{H}) ; 2.42(2 \mathrm{~s}, 3 \mathrm{H}) ; 4.10(\mathrm{~d}, 1 \mathrm{H}, J=$ 2.2); $4.15(\mathrm{~m}, 2 \mathrm{H}) ; 7.04(\mathrm{dd}, 2 \mathrm{H}, J=8.6,8.9) ; 7.90(\mathrm{dd}, 2 \mathrm{H}, J=5.4,8.8) .{ }^{13} \mathrm{C}-\mathrm{NMR}\left(75 \mathrm{MHz}, \mathrm{CDCl}_{3}\right): \delta 13.6,13.7,16.9$, 21.7, 26.2, 29.6, 61.7, 64.5, 77.2, 80.9, 81.3, 114.9 (d, $J=21.5), 131.2(\mathrm{~d}, J=8.8), 132.6,160.9,164.5(\mathrm{~d}, J=252), 167.6$, 169.7. ESIMS m/z $425\left(\left[\mathrm{M}+\mathrm{H}^{+}\right], \mathrm{C}_{21} \mathrm{H}_{29} \mathrm{FN}_{2} \mathrm{O}_{4} \mathrm{~S}\right.$ requires 424).

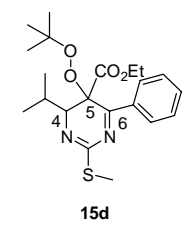

5-(tert-Butylperoxy)-5-ethoxycarbonyl-4-isopropyl-2-(methylthio)-6-phenyl-4,5-dihydropyrimidine (15d). ${ }^{1} \mathrm{H}-\mathrm{NMR}$ (300 $\left.\mathrm{MHz}, \mathrm{CDCl}_{3}\right): \delta 0.80(\mathrm{~d}, 3 \mathrm{H}, J=6.6) ; 1.04(\mathrm{~s}, 9 \mathrm{H}), 1.07$ (, 3H, $\left.J=7.2,7.1\right) ; 1.15(\mathrm{~d}, 3 \mathrm{H}, J=7.0) ; 1.91(\mathrm{~m}, 1 \mathrm{H}) ; 2.42(\mathrm{~s}$, $3 \mathrm{H}) ; 4.10(\mathrm{~m}, 1 \mathrm{H}) ; 4.11(\mathrm{~d}, 1 \mathrm{H}, J=3.4) ; 7.35(\mathrm{dd}, 2 \mathrm{H}, J=7.8,6.9) ; 7.43(\mathrm{dd}, 1 \mathrm{H}, J=7.4,7.1) ; 7.84(\mathrm{~d}, 2 \mathrm{H}, J=7.2) .{ }^{13} \mathrm{C}-$ NMR $\left(75 \mathrm{MHz}, \mathrm{CDCl}_{3}\right): \delta 13.5,13.7,17.0,21.7,26.2,29.6,61.6,64.5,80.9,81.1,127.4,128.8,130.8,136.5,161.0$, 167.6, 171.1. IR $(\mathrm{NaCl}) v_{\max } 1224,1566,1608,1746,3063 \mathrm{~cm}^{-1}$. ESIMS m/z $407.2\left(\left[\mathrm{M}+\mathrm{H}^{+}\right], \mathrm{C}_{21} \mathrm{H}_{30} \mathrm{~N}_{2} \mathrm{O}_{4} \mathrm{~S}\right.$ requires 406). HRMS $[\mathrm{M}+\mathrm{H}]^{+}: 407.1993$ (calc, Exact mass.: 407.2005).

The structure was determined based on evaluation of the HMQC and HMBC experiments to obtain connectivities between C4, C5 and C6. The 2-D spectral data are included in the other supplemental material. 
Below is our investigation of the application of the dehydrogenation procedure to a selection of heterocycles. As shown, oxazolines S-2a-b and thiazoline S-2c afforded variable amounts of desired oxazoles/thiazole S-5 (entries 1-3), accompanied by a number of side-products, which were currently assigned as compounds $\mathbf{S - 3}$ and $\mathbf{S - 4}$ (including their isomers) based on their LRMS and ${ }^{1} \mathrm{H}$ NMR data. These side -products appeared to be derived via the corresponding radical mechanism depicted in Figure $1 .^{12}$ In contrast, relatively clean dehydrogenation was observed with imidazoline $\mathbf{S}$ 2d (entry 4). The observation can be explained if the imidazolines, which contain a secondary N-H moiety, is the only substrate in this series that oxidizes with the mechanism shown in Figure 2. .

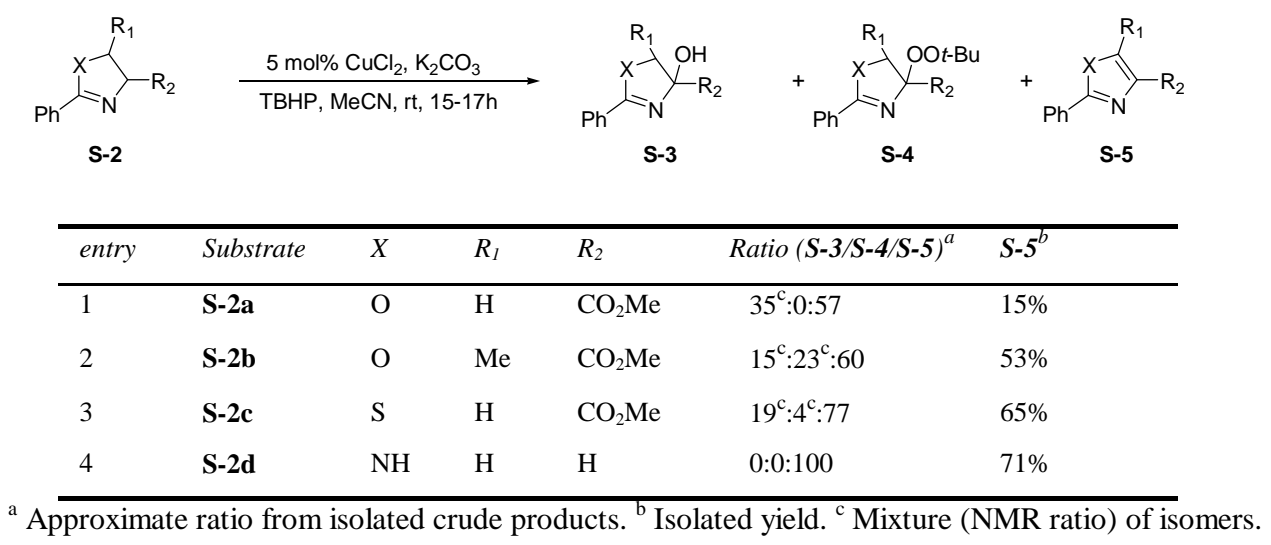

Heterocycles S-2a-c were prepared according to the literature procedure. ${ }^{13}$ Imidazoline $\mathbf{S - 2 d}$ was purchased from Aldrich Chemical and used as is.

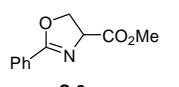

S-2a

4-Methoxycarbonyl-2-phenyl-4,5-dihydrooxazole (S-2a). ${ }^{13}$ Yield: $57 \%$. ${ }^{1} \mathrm{H}-\mathrm{NMR}\left(300 \mathrm{MHz}, \mathrm{CDCl}_{3}\right): \delta 3.79(\mathrm{~s}, 3 \mathrm{H})$; $4.56(\mathrm{dd}, 1 \mathrm{H}, J=8.9,10.4) ; 4.67(\mathrm{dd}, 1 \mathrm{H}, J=8.3,8.3) ; 4.93(\mathrm{dd}, 1 \mathrm{H}, J=8.9,10.5) ; 7.38(\mathrm{~m}, 2 \mathrm{H}) ; 7.47(\mathrm{~m}, 1 \mathrm{H}) ; 7.96(\mathrm{~d}$, $2 \mathrm{H}, J=8.1)$. ESIMS m/z $206\left(\left[\mathrm{M}+\mathrm{H}^{+}\right], \mathrm{C}_{11} \mathrm{H}_{11} \mathrm{NO}_{3}\right.$ requires 205).

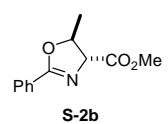

4-Methoxycarbonyl-5-methyl-2-phenyl-4,5-dihydrooxazole (S-2b). ${ }^{14}$ Yield: $31 \% .{ }^{1} \mathrm{H}-\mathrm{NMR}\left(300 \mathrm{MHz}, \mathrm{CDCl}_{3}\right): \delta 1.53$ $(\mathrm{d}, 3 \mathrm{H}, J=6.3) ; 3.80$ (s, 3H); 4.47 (d, 1H, $J=7.4) ; 4.99$ (m, 1H); 7.40 (m, 2H); $7.50(\mathrm{~m}, 1 \mathrm{H}) ; 7.99(\mathrm{~d}, 2 \mathrm{H}, J=7.1)$. ESIMS m/z $220\left(\left[\mathrm{M}+\mathrm{H}^{+}\right], \mathrm{C}_{12} \mathrm{H}_{13} \mathrm{NO}_{3}\right.$ requires 219).

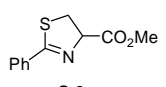

S-2c

\footnotetext{
${ }^{12}$ Dehydrogenation of oxazoles and thiazoles has been reported under analogous conditions that involve a radical mechanism. ${ }^{13}$

13 Meyers, A. I.; Tavares, F. X. J. Org. Chem. 1996, 61, 8207.

14 Watts, J.; Benn, A.; Flinn, N.; Monk, T.; Ramjee, M.; Ray, P.; Wang, Y.; Quibell, M. Bioorganic \& Medicinal Chemistry, 2004, 12, 2903.
} 
4-Methoxycarbonyl-2-phenyl-4,5-dihydrothiazole (S-2c). ${ }^{15}$ Yield: $53 \% .{ }^{1} \mathrm{H}-\mathrm{NMR}\left(300 \mathrm{MHz}, \mathrm{CDCl}_{3}\right): \delta 3.62(\mathrm{dd}, 1 \mathrm{H}, J$ $=9.5,11.3) ; 3.70(\mathrm{dd}, 1 \mathrm{H}, J=8.9,11.3) ; 3.82(\mathrm{~s}, 3 \mathrm{H}) ; 5.27(\mathrm{dd}, 1 \mathrm{H}, J=9.1,9.2) ; 7.40-7.44(\mathrm{~m}, 3 \mathrm{H}) ; 7.85(\mathrm{~d}, 2 \mathrm{H}, J=3.2)$. ESIMS m/z $222\left(\left[\mathrm{M}+\mathrm{H}^{+}\right], \mathrm{C}_{11} \mathrm{H}_{11} \mathrm{NO}_{2} \mathrm{~S}\right.$ requires 221).

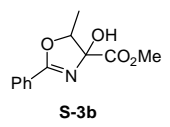

4-Hydroxy-4-methoxycarbonyl-5-methyl-2-phenyl-4,5-dihydrooxazole(S-3b). Obtained as $\sim 2.5: 1$ mixture. ${ }^{1} \mathrm{H}-\mathrm{NMR}$ (300 MHz, $\mathrm{CDCl}_{3}$ ): $\delta 143(\mathrm{~d}$, minor 3H, $J=6.8$ ); 1.49 (d, major 3H, $J=6.6$ ); 3.76 (s, minor 3H); 3.85 (s, major 3H); 4.77 (q, minor $1 \mathrm{H}, J=6.8) ; 5.10$ (q, major $1 \mathrm{H}, J=6.6) ; 7.42(\mathrm{~m}$, minor $2 \mathrm{H}+$ major $2 \mathrm{H}) ; 7.53(\mathrm{~m}$, minor $1 \mathrm{H}+\mathrm{major} 1 \mathrm{H}) ; 8.00$ (d, minor $2 \mathrm{H}+$ major $2 \mathrm{H}, 8.7)$. ESIMS m/z $236\left(\left[\mathrm{M}+\mathrm{H}^{+}\right], \mathrm{C}_{12} \mathrm{H}_{13} \mathrm{NO}_{4}\right.$ requires 235).

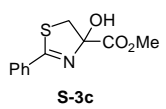

4-Hydroxy-4-methoxycarbonyl-2-phenyl-4,5-dihydrothiazole(S-3c). ${ }^{16}{ }^{1} \mathrm{H}-\mathrm{NMR}\left(300 \mathrm{MHz}, \mathrm{CDCl}_{3}\right): \delta 2.09$ (br s, $\left.1 \mathrm{H}\right)$; $3.70(\mathrm{~d}, 1 \mathrm{H}, J=12.5) ; 3.96(\mathrm{~s}, 3 \mathrm{H}) ; 4.08(\mathrm{~d}, 1 \mathrm{H}, J=12.4) ; 7.53(\mathrm{dd}, 2 \mathrm{H}, J=7.9,6.0) ; 7.67(\mathrm{~m}, 1 \mathrm{H}) ; 8.10(\mathrm{~d}, 2 \mathrm{H}, J=7.8)$. ESIMS m/z $238\left(\left[\mathrm{M}+\mathrm{H}^{+}\right], \mathrm{C}_{11} \mathrm{H}_{11} \mathrm{NO}_{3} \mathrm{~S}\right.$ requires 237$)$.

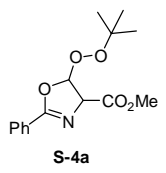

4-(tert-Butylhydroperoxy)-4-methoxycarbonyl-2-phenyl-4,5-dihydrooxazole (S-4a). Obtained as a mixture of isomers. The major isomer: ${ }^{1} \mathrm{H}-\mathrm{NMR}\left(300 \mathrm{MHz}, \mathrm{CDCl}_{3}\right): \delta 1.02(\mathrm{~s}, 9 \mathrm{H}) ; 3.64(\mathrm{~s}, 3 \mathrm{H}) ; 4.51(\mathrm{~d}, 1 \mathrm{H}, J=9.5) ; 4.65(\mathrm{~d}, 1 \mathrm{H}, J=9.2)$; $7.20(\mathrm{~m}, 3 \mathrm{H}) ; 7.83(\mathrm{~d}, 2 \mathrm{H}, J=7.4)$. ESIMS m/z $294\left(\left[\mathrm{M}+\mathrm{H}^{+}\right], \mathrm{C}_{15} \mathrm{H}_{19} \mathrm{NO}_{5}\right.$ requires 293).

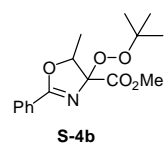

4-(tert-Butylhydroperoxy)-4-methoxycarbonyl-5-methyl-2-phenyl-4,5-dihydrooxazole(S-4b). Obtained as a $~ 2: 1$ mixture of isomers. ${ }^{1} \mathrm{H}-\mathrm{NMR}\left(300 \mathrm{MHz}, \mathrm{CDCl}_{3}\right.$ ): $\delta 1.23$ (s, major 9H); 1.24 (s, minor 3H); 1.35 (d, major 3H, $J=6.7$ ); $1.59(\mathrm{~d}$, minor $3 \mathrm{H}, J=6.8$ ); 3.83 (major 3H); 3.93 (minor 3H); 4.79 (q, major $1 \mathrm{H}, J=6.8$ ); 5.09 (q, minor $1 \mathrm{H}, J=6.8$ ); 7.45 (m, major $3 \mathrm{H}+$ minor $3 \mathrm{H}) ; 8.04(\mathrm{~m}$, major $2 \mathrm{H}+$ minor $2 \mathrm{H})$. ESIMS m/z $308\left(\left[\mathrm{M}+\mathrm{H}^{+}\right], \mathrm{C}_{16} \mathrm{H}_{21} \mathrm{NO}_{5}\right.$ requires 307$)$.

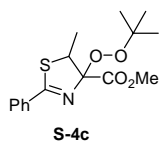

4-(tert-Butylhydroperoxy)-4-methoxycarbonyl-2-phenyl-4,5-dihydrothiazole (S-4c). Obtained as a mixture of isomers. The major isomer: ${ }^{1} \mathrm{H}-\mathrm{NMR}\left(300 \mathrm{MHz}, \mathrm{CDCl}_{3}\right): \delta 1.25(\mathrm{~s}, 9 \mathrm{H}) ; 3.82(\mathrm{~d}, 1 \mathrm{H}, J=12.6) ; 3.87(\mathrm{~s}, 3 \mathrm{H}) ; 4.06(\mathrm{~d}, 1 \mathrm{H}, J=$ 12.6); 7.41-7.53 (m, 3H); $7.94(\mathrm{~d}, 2 \mathrm{H}, J=7.2)$. ESIMS m/z $310\left(\left[\mathrm{M}+\mathrm{H}^{+}\right], \mathrm{C}_{15} \mathrm{H}_{19} \mathrm{NO}_{4} \mathrm{~S}\right.$ requires 309).

15 Wuts, P. G. M.; Northuis, J. M.; Kwan, T. A. J. Org. Chem., 2000, 65, 9223.

16 Waldemar, A.; Ehrig, V. Synthesis, 1976, 12, 817. 
S-5a

4-Methoxycarbonyl-2-(phenyl)oxazole (S-5a). ${ }^{13} \quad{ }^{1} \mathrm{H}-\mathrm{NMR}\left(300 \mathrm{MHz}, \mathrm{CDCl}_{3}\right): \delta 3.95$ (s, 3H); 7.49 (m, 3H); $8.12(\mathrm{~m}$, $2 \mathrm{H}) ; 8.29(\mathrm{~s}, 1 \mathrm{H})$. ESIMS m/z $204\left(\left[\mathrm{M}+\mathrm{H}^{+}\right], \mathrm{C}_{11} \mathrm{H}_{9} \mathrm{NO}_{3}\right.$ requires 203).

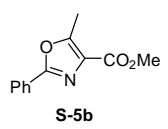

4-Methoxycarbonyl-5-methyl-2-(phenyl)oxazole (S-5b). ${ }^{17}{ }^{1} \mathrm{H}-\mathrm{NMR}\left(300 \mathrm{MHz}, \mathrm{CDCl}_{3}\right): \delta 1.02$. ESIMS m/z $218([\mathrm{M}+$ $\left.\mathrm{H}^{+}\right], \mathrm{C}_{12} \mathrm{H}_{11} \mathrm{NO}_{3}$ requires 217).

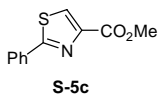

4-Methoxycarbonyl-2-(phenyl)thiazole (S-5c). ${ }^{18} \quad{ }^{1} \mathrm{H}-\mathrm{NMR}\left(300 \mathrm{MHz}, \mathrm{CDCl}_{3}\right): \delta 3.97(\mathrm{~s}, 3 \mathrm{H}) ; 7.45$ (m, 3H); 8.00 (m, $1 \mathrm{H}) ; 8.17(\mathrm{~s}, 1 \mathrm{H})$. ESIMS m/z $220\left(\left[\mathrm{M}+\mathrm{H}^{+}\right], \mathrm{C}_{11} \mathrm{H}_{9} \mathrm{NO}_{2} \mathrm{~S}\right.$ requires 219).

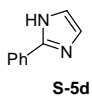

2-(phenyl)imidazole (S-5d). ${ }^{19}{ }^{1} \mathrm{H}-\mathrm{NMR}\left(300 \mathrm{MHz}, \mathrm{CDCl}_{3}\right): \delta 7.13(\mathrm{~s}, 2 \mathrm{H}) ; 7.32(\mathrm{~m}, 3 \mathrm{H}) ; 7.89(\mathrm{~m}, 2 \mathrm{H}) ; 10.9(\mathrm{br} \mathrm{s}, 1 \mathrm{H})$. ESIMS m/z $203\left(\left[\mathrm{M}+\mathrm{H}^{+}\right], \mathrm{C}_{11} \mathrm{H}_{10} \mathrm{~N}_{2} \mathrm{O}_{2}\right.$ requires 202).

Clapham, B.; Spanka, C.; Janda, K. D., Org. Lett., 2001, 3, 2173.

18 Waldemar, A.; Ehrig, V. Synthesis, 1976, 12, 817.

19 Highey, J. L.; Knapp, S.; Schugar, H. Synthesis, 1980, 489. 